\title{
Secretagogin expression in the vertebrate brainstem with focus on the noradrenergic system and implications for Alzheimer's disease
}

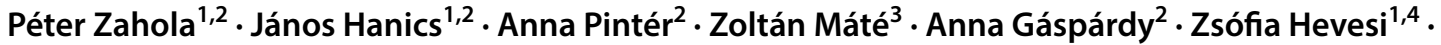 \\ Diego Echevarria ${ }^{5}$. Csaba Adori ${ }^{6}$. Swapnali Barde ${ }^{6} \cdot$ Beáta Töröcsik $^{7}$. Ferenc Erdélyi ${ }^{3}$. Gábor Szabó ${ }^{3}$. \\ Ludwig Wagner $^{8} \cdot$ Gabor G. Kovacs $^{9} \cdot$ Tomas Hökfelt $^{6} \cdot$ Tibor Harkany $^{4,6} \cdot$ Alán Alpár $^{1,2}$ (])
}

Received: 20 November 2018 / Accepted: 3 May 2019 / Published online: 29 May 2019

(c) The Author(s) 2019

\begin{abstract}
Calcium-binding proteins are widely used to distinguish neuronal subsets in the brain. This study focuses on secretagogin, an EF-hand calcium sensor, to identify distinct neuronal populations in the brainstem of several vertebrate species. By using neural tube whole mounts of mouse embryos, we show that secretagogin is already expressed during the early ontogeny of brainstem noradrenaline cells. In adults, secretagogin-expressing neurons typically populate relay centres of special senses and vegetative regulatory centres of the medulla oblongata, pons and midbrain. Notably, secretagogin expression overlapped with the brainstem column of noradrenergic cell bodies, including the locus coeruleus (A6) and the A1, A5 and A7 fields. Secretagogin expression in avian, mouse, rat and human samples showed quasi-equivalent patterns, suggesting conservation throughout vertebrate phylogeny. We found reduced secretagogin expression in locus coeruleus from subjects with Alzheimer's disease, and this reduction paralleled the loss of tyrosine hydroxylase, the enzyme rate limiting noradrenaline synthesis. Residual secretagogin immunoreactivity was confined to small submembrane domains associated with initial aberrant tau phosphorylation. In conclusion, we provide evidence that secretagogin is a useful marker to distinguish neuronal subsets in the brainstem, conserved throughout several species, and its altered expression may reflect cellular dysfunction of locus coeruleus neurons in Alzheimer's disease.
\end{abstract}

Keywords Alzheimer's disease $\cdot$ Calcium-binding proteins $\cdot$ Locus coeruleus $\cdot$ Norepinephrine $\cdot$ Phylogenetic conservation

Electronic supplementary material The online version of this article (https://doi.org/10.1007/s00429-019-01886-w) contains supplementary material, which is available to authorized users.

Alán Alpár

Alpar.Alan@med.semmelweis-univ.hu

1 SE NAP B Research Group of Experimental Neuroanatomy and Developmental Biology, Semmelweis University, Budapest, Hungary

2 Department of Anatomy, Semmelweis University, Budapest, Hungary

3 Institute of Experimental Medicine, Hungarian Academy of Sciences, Budapest, Hungary

4 Department of Molecular Neurosciences, Center for Brain Research, Medical University of Vienna, 1090 Vienna, Austria

\section{Introduction}

Our brainstem harbours a wealth of distinctly or ambiguously demarcated cell groups with various functions serving basic physiological needs. Pathways of special senses have relay centres in the cranial brainstem: the superior colliculus

5 Institute of Neuroscience, University of Miguel Hernandez de Elche, Alicante, Spain

6 Department of Neuroscience, Karolinska Institutet, Biomedicum 7D, SE-17165 Stockholm, Sweden

7 Department of Medical Biochemistry, Semmelweis University, Budapest, Hungary

8 Department of Internal Medicine III, Medical University of Vienna, Vienna, Austria

9 Institute of Neurology, Medical University of Vienna, Vienna, Austria 
and the lateral geniculate nucleus of the visual system, and the inferior colliculus and the medial geniculate nucleus of the acoustic system are situated in the mesencephalon. Cranial nerve nuclei of different modalities are arranged in logical mediolateral and craniocaudal orders, reaching from the caudal medulla oblongata to the cranial midbrain. These include stations of specific sensory pathways, like the cochlear and vestibular nuclei in the caudal pons, or complex vegetative centres, like the solitary tract nucleus in the medulla oblongata. Other autonomic integrative centres, like the periaqueductal grey involved in behavioural responses to threatening stimuli (Faull et al. 2019) and opioid modulation of pain (Martins and Tavares 2017), or the parabrachial nuclear complex controlling fluid and food homoeostasis, cardiovascular functions (Davern 2014) and body temperature (Morrison and Nakamura 2011) appear as independent nuclei in the brainstem. Activating systems using biogenic amines as neurotransmitters have their origin in the brainstem: noradrenaline- (A1-A6 fields), adrenaline- (C1-C3 fields) or serotonin-containing (B1-B8 fields) neurons from nuclei, and their projections richly innervate many forebrain regions, the cerebellum and the spinal cord. The nuclei and centres of these different systems (Fig. 1) are intertwined and may in the brainstem even overlap, which has made their identification and separation an ongoing challenge.

The classical neuroanatomical toolbox including, e.g., ubiquitous neuronal staining or metal-based impregnations, was the first pioneering step to identify major brainstem centres. The advent of tract tracing and immunohistochemistry opened a new dimension in understanding the layout of brainstem circuits. Researchers not only identified hitherto unknown nuclei, but were able to demarcate subregions, further unravelling cellular heterogeneity within cell groups previously thought to be homogenous (Dahlstroem and Fuxe 1964; Ljungdahl et al. 1978; Markia et al. 2008; Palkovits 1999; Zaborszky et al. 1984). Although chemogenetic and optogenetic tools brought a quantum leap in the functional classification of neurons, neurochemical markers remain of foremost importance to identify neuronal populations in the brain (Rees et al. 2017), with calcium-binding proteins (CaBPs) being common-choice candidates to distinguish cell types or nuclei (Andressen et al. 1993; Freund and Buzsáki 1996; Riedel et al. 2002).

Activation of signalling pathways is a typical calcium $\left(\mathrm{Ca}^{2+}\right)$-dependent mechanism with $\mathrm{Ca}^{2+}$-sensor proteins specifying downstream protein-protein interactions (Skelton et al. 1994). Secretagogin, a $\mathrm{Ca}^{2+}$-sensor protein (Wagner et al. 2000), undergoes conformational changes upon $\mathrm{Ca}^{2+}$ binding (Rogstam et al. 2007) to primarily affect protein turnover and exocytosis (Gartner et al. 2007). In agreement with its involvement in the exocytotic machinery, secretagogin, found in the mammalian rodent and non-primate
(Hanics et al. 2017; Mulder et al. 2009, 2010) and human (Attems et al. 2012a) brain and spinal cord (Zhang et al. 2016), has been associated with presynaptic neurotransmitter release (Romanov et al. 2015; Zhang et al. 2016). Nevertheless, while forebrain distribution of secretagoginexpressing $^{(+)}$neurons is well characterized (Alpar et al. 2012; Attems et al. 2008; Garas et al. 2016; Gyengesi et al. 2013; Kosaka and Kosaka 2013; Kosaka et al. 2017; Mulder et al. 2009; Romanov et al. 2015), the localization and phenotype of secretagogin ${ }^{+}$neurons in the brainstem remain elusive.

Here, we use secretagogin as a valuable neuroanatomical marker to identify brainstem nuclei in vertebrates, including avian, rodent and human brains. In addition to relay centres of special senses and vegetative regulatory centres, we classify the brainstem noradrenaline stress axis as a focus of secretagogin expression in the murine and human brain. Noradrenergic neurons, assembled in a column of nuclei throughout the medullary and pontine brainstem (Dahlstroem and Fuxe 1964), are extensively connected to cortical and subcortical forebrain regions to orchestrate central responses to, e.g. stress (Aston-Jones et al. 1996; Itoi and Sugimoto 2010; Samuels and Szabadi 2008a, b). Imbalance in noradrenaline function may result in affective, panic and anxiety disorders (Bremner et al. 1996a, b; Charney 2003; Kvetnansky et al. 2009; Samuels and Szabadi 2008a, b), representing serious and costly morbidities and a burden on public health worldwide (Alloul et al. 1998; Takizawa et al. 2015). Of note, locus coeruleus is critically vulnerable already in the initial phase of neurodegenerative diseases, most notably Alzheimer's disease (Braak and Del Tredici 2012; Tomlinson et al. 1981). We suggest that altered secretagogin expression in locus coeruleus neurons is a clinicopathological sign of Alzheimer's disease paralleling or even preceding tyrosine hydroxylase $(\mathrm{TH})$ loss.

\section{Results}

\section{Secretagogin is expressed in the locus coeruleus of the early rhombencephalic neural tube}

We have isolated and prepared neural tube whole mounts from early mouse embryos to determine the onset of secretagogin expression in the foetal brainstem (Fig. 2). At embryonic day 8.5 (E8.5), no secretagogin immunoreactivity could be detected in any rhombencephalic domain (Fig. 2a). Brainstem neurons began to express secretagogin by E11.5, with a "hot spot" in the caudal midbrain (Fig. 2b) and in cell contingents along the basal plate and limiting sulcus of the medulla oblongata and the cervical flexure. At E11.5, TH expression was detected in a cell population dispersed 
Fig. 1 Distribution of secretagogin in the rat brainstem I. Secretagogin ${ }^{+}$somata were identified in coronal sections throughout the rat brainstem. Low-power micrographs are paired with schemata of coronal brain sections to indicate craniocaudal levels. Secretagogin ${ }^{+}$ neurons-containing regions are indicated in purple. Superior colliculus, the microcellular tegmental nucleus, the dorsal nucleus of vagus and the noradrenergic cell groups including A1, A5, A6 and A7 typically expressed secretagogin. For high-power images, please see Fig. 4. A1, A5, A7 noradrenergic cell groups, $C l$ adrenergic cell group, $C n$ cuneiform nucleus, DLPAG dorsolateral periaqueductal grey, $I C$ inferior colliculus, $I P$ interpeduncular nucleus, $i R T$ intermediate reticular nucleus, $L C$ locus coeruleus, $L L$ lateral lemniscus, $L P M C$ lateral posterior thalamic nucleus mediocaudal part, MiTg microcellular tegmental nucleus, $m R T$ mesencephalic vestibular nucleus, $N T S$ solitary tract nucleus, $P B$ parabrachial nucleus, $P P$ peripeduncular nucleus, $P$ tg pedunculopontine tegmental nucleus, $s B$ subbrachial nucleus, $S C$ superior colliculus, $s p 5$ spinal tract nucleus of $\mathrm{V}, V L P A G$ ventrolateral periaqueductal grey, $X$ dorsal nucleus of $X$ reticular formation, $M V$ medial
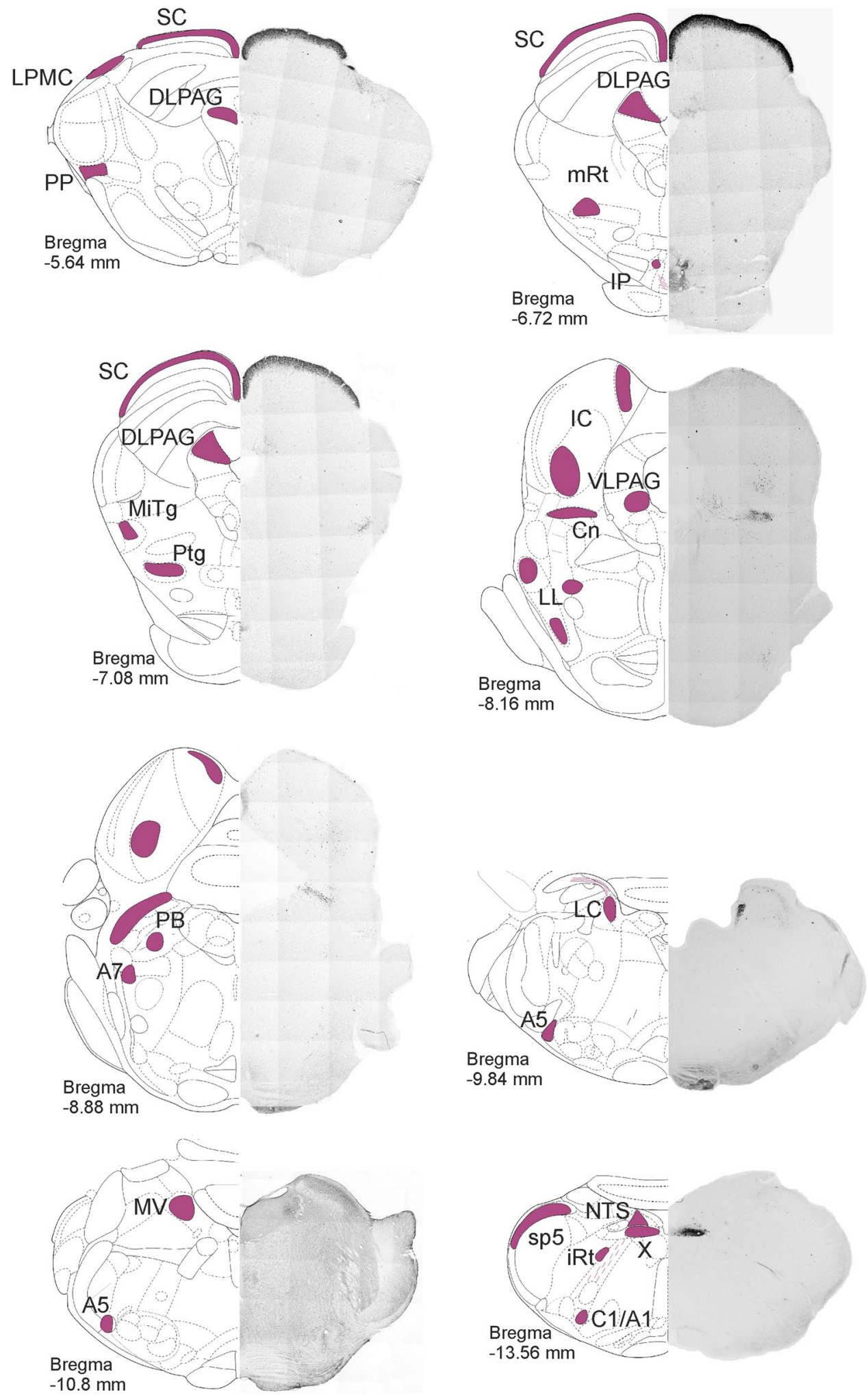

throughout the medulla oblongata and pons. Those $\mathrm{TH}^{+}$ cells that had concentrated in the locus coeruleus (Fig. 2b, $b_{1}$ ) were secretagogin immunoreactive (Fig. $2 b_{1}{ }_{1}, b_{1}{ }_{1}$ ') with $\mathrm{TH}^{+} /$secretagogin ${ }^{-}$cells being the exception rather than the rule. We conclude that secretagogin and $\mathrm{TH}$ are expressed during mouse ontogeny. coincidentally in the major noradrenergic brainstem centre 


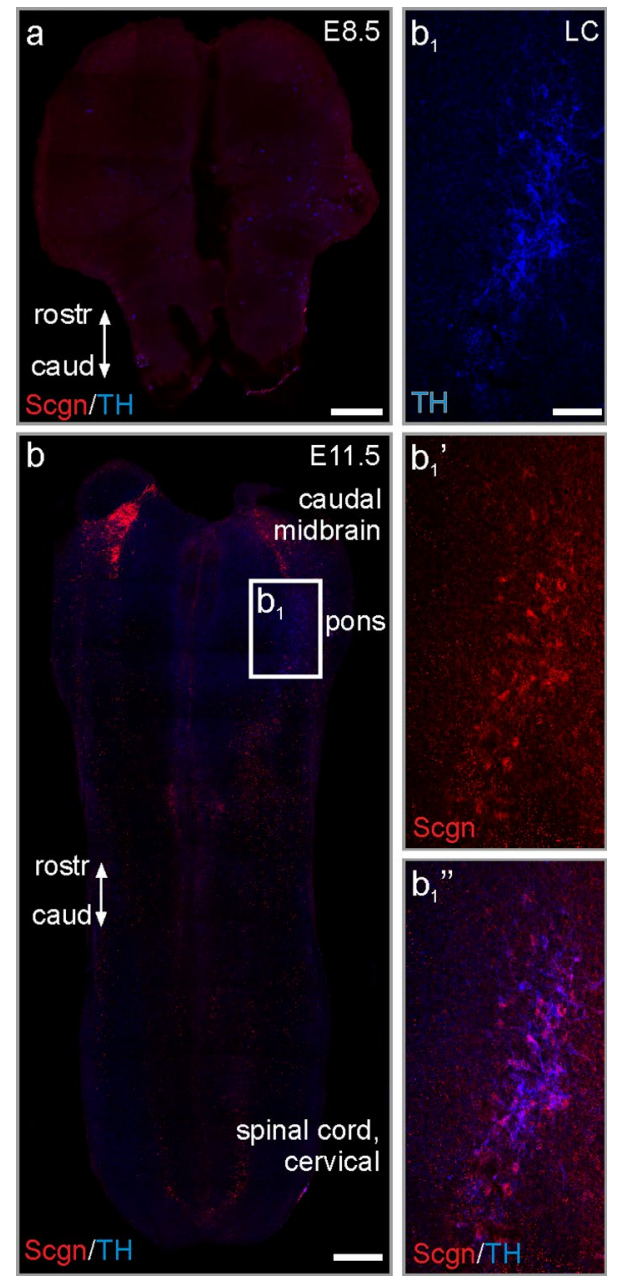

Fig. 2 Secretagogin expression in the early neural tube. a Neural tube whole mount from E8.5 mouse embryos lacks secretagogin immunoreactivity. b Secretagogin expression appears at E11.5 in the caudal midbrain. $\mathbf{b}_{\mathbf{1}}-\mathbf{b}_{\mathbf{1}}$ " $\mathrm{TH}^{+}$neurons share secretagogin immunoreactivity in the locus coeruleus. caud caudal, rostr rostral, Scgn secretagogin, TH tyrosine hydroxylase. Scale bars $300 \mu \mathrm{m}(\mathbf{a}, \mathbf{b}), 60 \mu \mathrm{m}\left(\mathbf{b}_{1}\right)$

\section{Distribution of secretagogin ${ }^{+}$neurons in the rodent brainstem}

Our systematic survey through the rat brainstem revealed several medullary, pontine and midbrain domains which harboured secretagogin ${ }^{+}$neurons (Fig. 1). In sagittal sections, i.a. the superior colliculus, the parabrachial nucleus and the A5 field emerged as secretagogin 'hot spots' (Fig. 3). Using serial coronal sections of the brainstem (Fig. 1), we identified secretagogin immunoreactivity in the superior colliculus and in the dorsolateral part of the periaqueductal grey in the midbrain (Fig. 4a-a ${ }_{1}$ "), the locus coeruleus, parabrachial nucleus in the pons (Fig. 4b, c), and the dorsal nucleus of vagus in the medulla oblongata (Fig. $4 \mathrm{~d}_{1}, \mathrm{~d}_{1}{ }^{\text {'). We identified }}$ identical distributions of immunoreactive cells when using our primary antibodies produced either in rabbit or goat, although the goat antiserum was associated with higher tissue background (Fig. 4a, a 1 , b, b 1 , d, d d $_{1}$. In addition, several smaller groups of secretagogin ${ }^{+}$neurons appeared in the medulla oblongata and pons, which were identified as the A1 (Fig. 4e), A5 and A7 fields. Secretagogin/TH double immunolabelling in the locus coeruleus (Fig. 4f-f'") showed that $75.5 \pm 6.9 \%$ (average \pm SEM) of all labelled cells coexpressed these markers, leaving $23.3 \pm 2.2 \%$ and $1.1 \pm 0.9 \%$ of cells single labelled for $\mathrm{TH}$ and secretagogin, respectively. The processes of secretagogin ${ }^{+}$locus coeruleus neurons were typically directed towards the ventricular space, likely representing dendrites (Fig. $4 \mathrm{f}_{1}$ ). At the same time, $\mathrm{TH}^{+}$ neurons in the ventral tegmentum of the midbrain did not co-express secretagogin (Fig. S1a-a"). Likewise, serotonin ${ }^{+}$ median raphe neurons were also immunonegative for secretagogin (Fig. S1b-b"). Furthermore, in the ventral part of the periaqueductal grey, where serotonin ${ }^{+}$and secretagogin ${ }^{+}$ neurons coincidentally occurred, we could not identify their co-expression either (Fig. S1c-c').
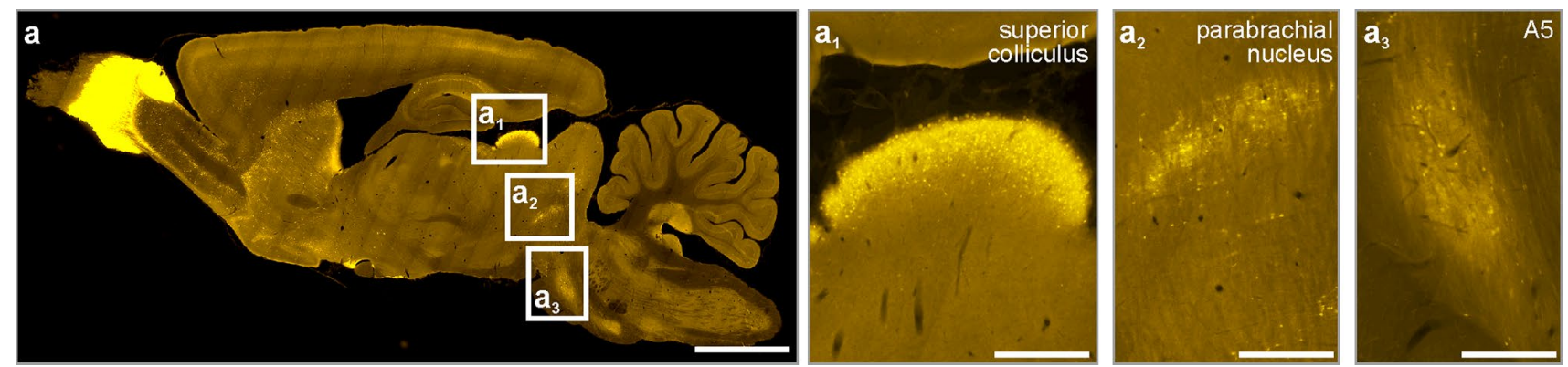

Fig. 3 Distribution of secretagogin in the rat brainstem II. Sagittal sections of the rat brain revealed superior colliculus ( $\left.\mathbf{a}_{1}\right)$, the parabrachial nucleus $\left(\mathbf{a}_{2}\right)$ and the A5 field $\left(\mathbf{a}_{3}\right)$ as typical loci which harbour secretagogin ${ }^{+}$neurons. Scale bars $1 \mathrm{~mm}(\mathbf{a}), 300 \mu \mathrm{m}\left(\mathbf{a}_{1}, \mathbf{a}_{2}, \mathbf{a}_{3}\right)$ 

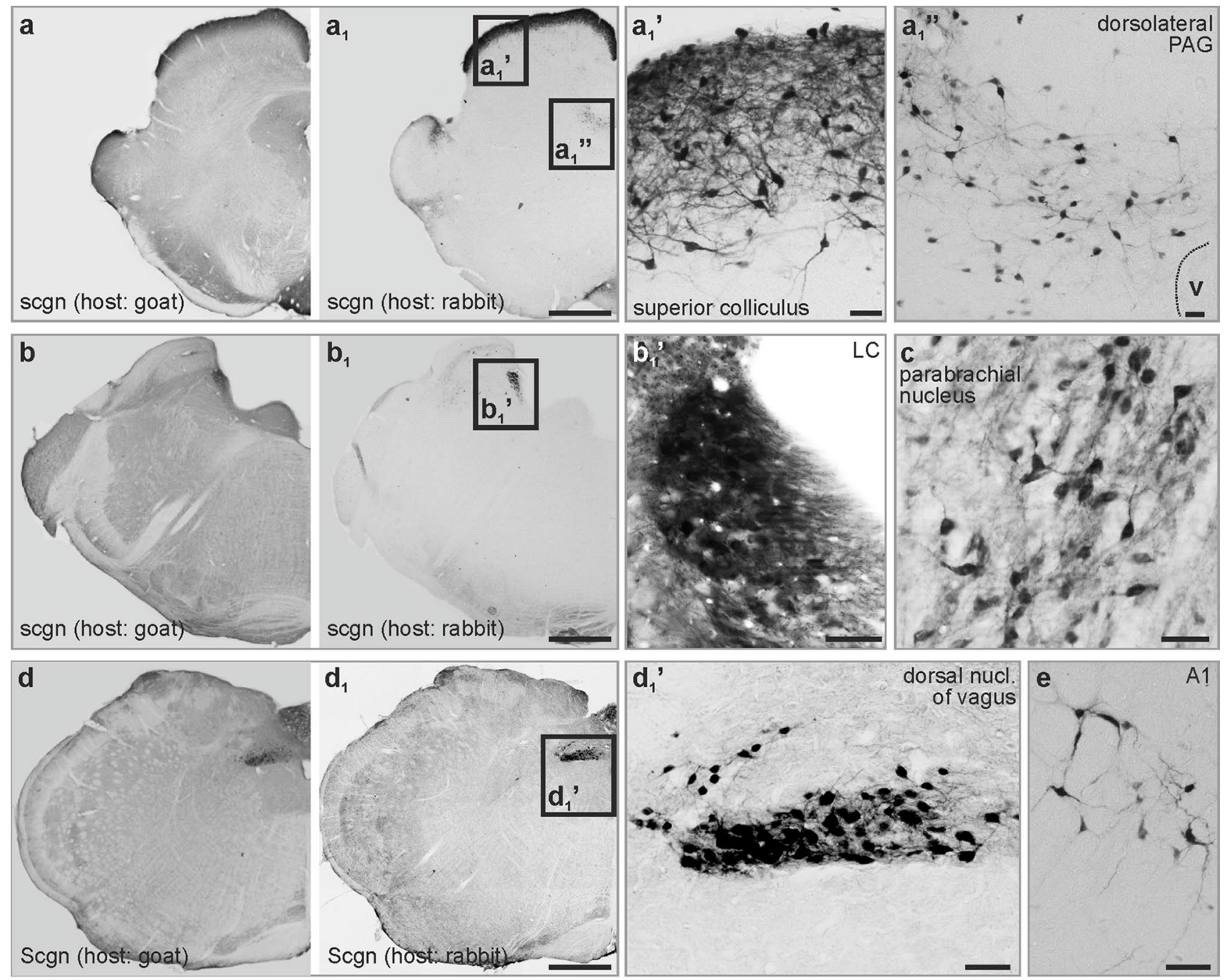

e
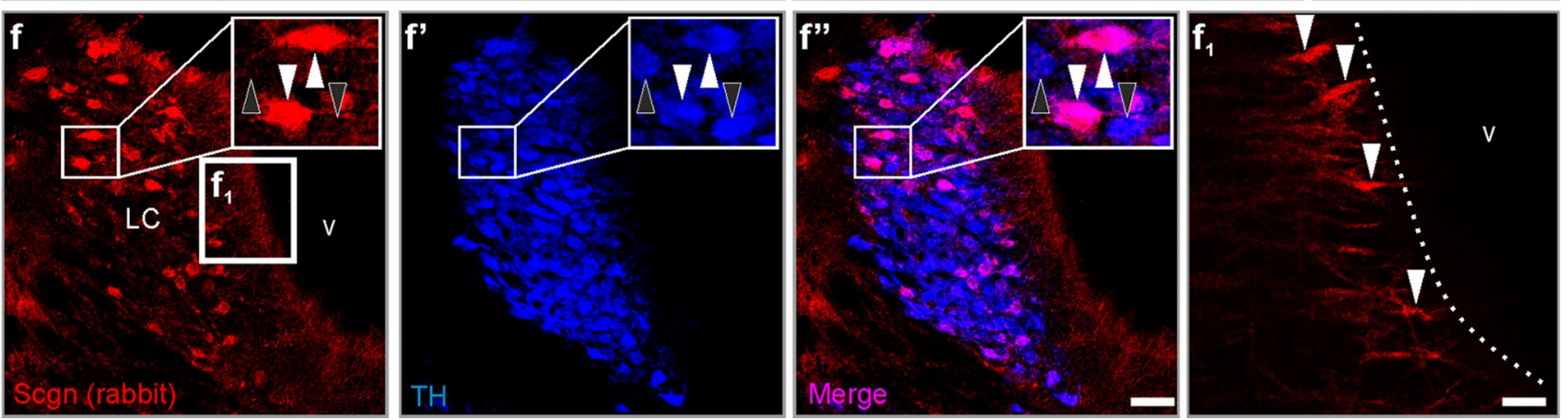

Fig. 4 Distribution of secretagogin in the rat brainstem III. (a-a, ") In the midbrain, secretagogin ${ }^{+}$neurons condensed in the superior col-

in $\mathrm{TH}^{+}$neurons in the locus coeruleus (white arrowheads indicate $\mathrm{TH}^{+} /$secretagogin ${ }^{+}$neurons, black arrowheads point to $\mathrm{TH}^{+} /$secretagogin $^{-}$neurons). $\mathbf{f}_{1}$ The processes of secretagogin ${ }^{+}$locus coeruleus neurons reached the ventricular surface (white arrowheads). $L C$ locus coeruleus, scgn secretagogin, $P A G$ periaqueductal grey. Scale bars $1 \mathrm{~mm}\left(\mathbf{a}_{1}, \mathbf{b}_{1}, \mathbf{d}_{1}\right), 100 \mu \mathrm{m}\left(\mathbf{a}_{\mathbf{1}}{ }^{\prime}\right), 40 \mu \mathrm{m}\left(\mathbf{a}_{\mathbf{1}}, \mathbf{b}_{\mathbf{1}}{ }^{\prime}\right), 30 \mu \mathrm{m}\left(\mathbf{c}, \mathbf{d}_{\mathbf{1}}{ }^{\prime}, \mathbf{e}, \mathbf{f}^{\prime}{ }^{\prime}\right)$, $10 \mu \mathrm{m}\left(\mathbf{a}_{\mathbf{1}}, \boldsymbol{\prime}\right), 3 \mu \mathrm{m}\left(\mathbf{f}_{\mathbf{1}}\right)$ 


\section{Relation of secretagogin to other CaBPs}

CaBPs are routinely used to typify neurons in the brain (Skelton et al. 1994). They exhibit largely non-overlapping expression patterns (Andressen et al. 1993; Freund and Buzsáki 1996) with occasional co-localization in the olfactory circuit, ventral pallidum and renewing cells of the dentate gyrus (del Rio and DeFelipe 1997; Wouterlood et al. 2001). In the brainstem, secretagogin ${ }^{+}$neurons form largely non-overlapping populations with only occasional, domain-specific overlap/co-expression of CaBPs in select nuclei (Fig. 5): in the dorsal nucleus of vagus and commissural part of the solitary tract nucleus, a complementary expression pattern of calretinin and secretagogin was shown (Fig. 5a-a," ${ }_{1}$ ), with many calretinin ${ }^{+} /$secretagogin $^{+}$ double-labelled neurons in the dorsolateral part of the solitary tract nucleus at the same level (Fig. 5c- $\mathrm{c}_{1}$,"'). By using simultaneous labelling for secretagogin/calretinin/parvalbumin, $68.5 \pm 5.4 \%$ of all secretagogin ${ }^{+}$neurons co-expressed calretinin. Secretagogin/parvalbumin co-expression was exceptionally rare: we only identified a single secretagogin ${ }^{+}$ neuron which also harboured parvalbumin (Fig. 5c ${ }_{1}{ }^{\prime}, \mathrm{c}_{1}{ }^{\prime}$ ). We found a diverse co-expression pattern in the spinal tract nucleus of the trigeminus: using secretagogin/calretinin/ parvalbumin or secretagogin/calbindin D28k/parvalbumin immunohistochemistry, secretagogin often co-localized with calbindin D28k (Fig. $5 \mathrm{e}-\mathrm{e}_{2}, 40.3 \pm 15.9 \%$ of all secretagogin $^{+}$neurons co-expressed calbindin D28k), only sporadically with calretinin (Fig. $5 b-b_{2}, 2.8 \pm 3.2 \%$ of all secretagogin ${ }^{+}$neurons co-expressed calretinin), but not with parvalbumin (Fig. 5b-b ${ }_{1}{ }^{\prime}$, e-e $e_{2}$ ). Similarly, secretagogin ${ }^{+}$ neurons were immunonegative for parvalbumin in the ventrolateral medulla (Fig. 5d), as well as in the parabrachial nucleus (Fig. 5f). Secretagogin ${ }^{+}$neurons in the parabrachial nucleus did not share calretinin (Fig. $5 \mathrm{f}-\mathrm{f}_{1}$ ") or calbindin immunoreactivity (Fig. 5g), either. We acknowledge that the above proportions were calculated using an investigative method and not by stereology; thus, it provides approximate instead of accurate results regarding cell numbers/ proportions.

\section{Secretagogin in the mouse and avian brainstem}

We next asked if secretagogin expression exhibited quasiequivalent distribution in different vertebrates. To this end, serial sections of the mouse, chick and human brainstem were analysed for secretagogin ${ }^{+}$neurons to see if an evolutionary continuum may exist among vertebrates. First, we generated a mouse line which expressed regulatory elements of the secretagogin promoter fused with EGFP on an artificial bacterial chromosome ( $\left.S c g n^{\text {BAC/egfp }}\right)$ to control for and validate our immunoreagents. We found extensive overlap between EGFP expression and secretagogin immunoreactivity (Fig. 6a-a,", Fig. S2) in brainstem nuclei (Table S1). The lack of secretagogin immunoreactivity in $\mathrm{EGFP}^{+}$cells was attributed to low protein abundance in $\mathrm{EGFP}^{+}$neurons, i.e. below immunocytochemical detectability (in our hands), because there was no hindbrain area in which the complete segregation of EGFP, suggesting ectopic expression, was seen. The distribution pattern of immunoreactive cells was largely similar to what we found in rats: secretagogin $^{+}$neurons typically occurred in the microcellular tegmental nucleus (Fig. 6a-a ${ }_{1}$ ', d, $d_{1}$ ), nucleus of the solitary tract $\left(6 b, b_{1}\right)$, the dorsal nucleus of vagus (Fig. $6 c$, $c_{1}$ ), the noradrenergic fields, especially locus coeruleus (Fig. 6e), and the superior colliculus (Fig. 6f, $f_{1}$ ). Yet, we detected differences between the two species: in the dorsolateral part of the periaqueductal grey, where immunoreactive somata were found in rat but only immunoreactive fibres and terminals in the mouse brain (Fig. S3a, a'). In rats, the interpeduncular nucleus contained secretagogin ${ }^{+}$cell bodies in its dorsal versus secretagogin ${ }^{+}$cell bodies and fibres in its lateral division: no such immunoreactivity was detected in the corresponding region of mice (Fig. S3b, b'). Similarly, no immunoreactivity was detected in the inferior colliculus (Fig. S3c, c') and medial vestibular nucleus (Fig. S3d, d') of mice.

\section{Secretagogin in the domestic chicken}

CaBPs are molecules whose structures show significant evolutionary conservation (Andressen et al. 1993). Neuronal subtypes of select brain areas typically retain their $\mathrm{CaBP}$ expression profiles across vertebrate species (Andressen et al. 1993; Gati et al. 2014). The brainstem harbours vegetative centres and nuclei, which share similarity, or even identity, in both lower and higher order vertebrates. We selected the domestic chick (Gallus domesticus) as a precocial avian species with immediate maturity after hatching to verify our hypothesis that secretagogin has an evolutionary preserved neuron-specific distribution in the vertebrate brainstem. Thus, the vagal nucleus (Fig. 7a, $\mathrm{a}_{1}$ ), the ventrolateral medulla (Fig. $7 b, b_{1}$ ), the vestibular nuclei and the locus coeruleus (Fig. 7c), midbrain nuclei like the ventral tegmental area and periaqueductal grey (Fig. $7 \mathrm{~d}-\mathrm{d}_{2}$ ) were typical secretagogin-expressing foci. Of note, we found secretagogin ${ }^{+}$neurons in areas which cannot be directly correlated to mammalian brain structures. Thus, the supraspinal nucleus-innervating the upper neck muscles, but being separate from the nucleus where the accessory nerve arises (Wild 1981) - also contained immunoreactive cells (not shown) and smaller islets of secretagogin ${ }^{+}$neurons were identified in different subdivisions of the reticular formation, especially in the midbrain (Fig. 7e). In conclusion, secretagogin is an evolutionarily conserved protein in many vertebrate brainstem nuclei. 

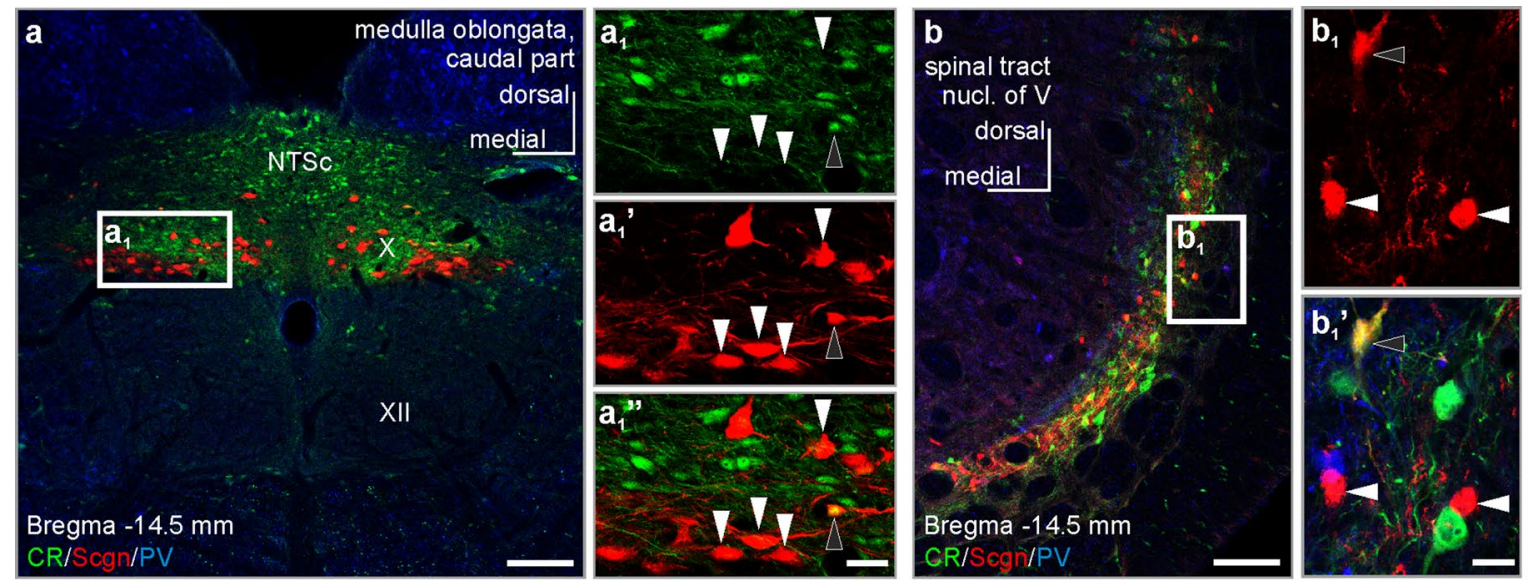

$\mathbf{b}_{2}$
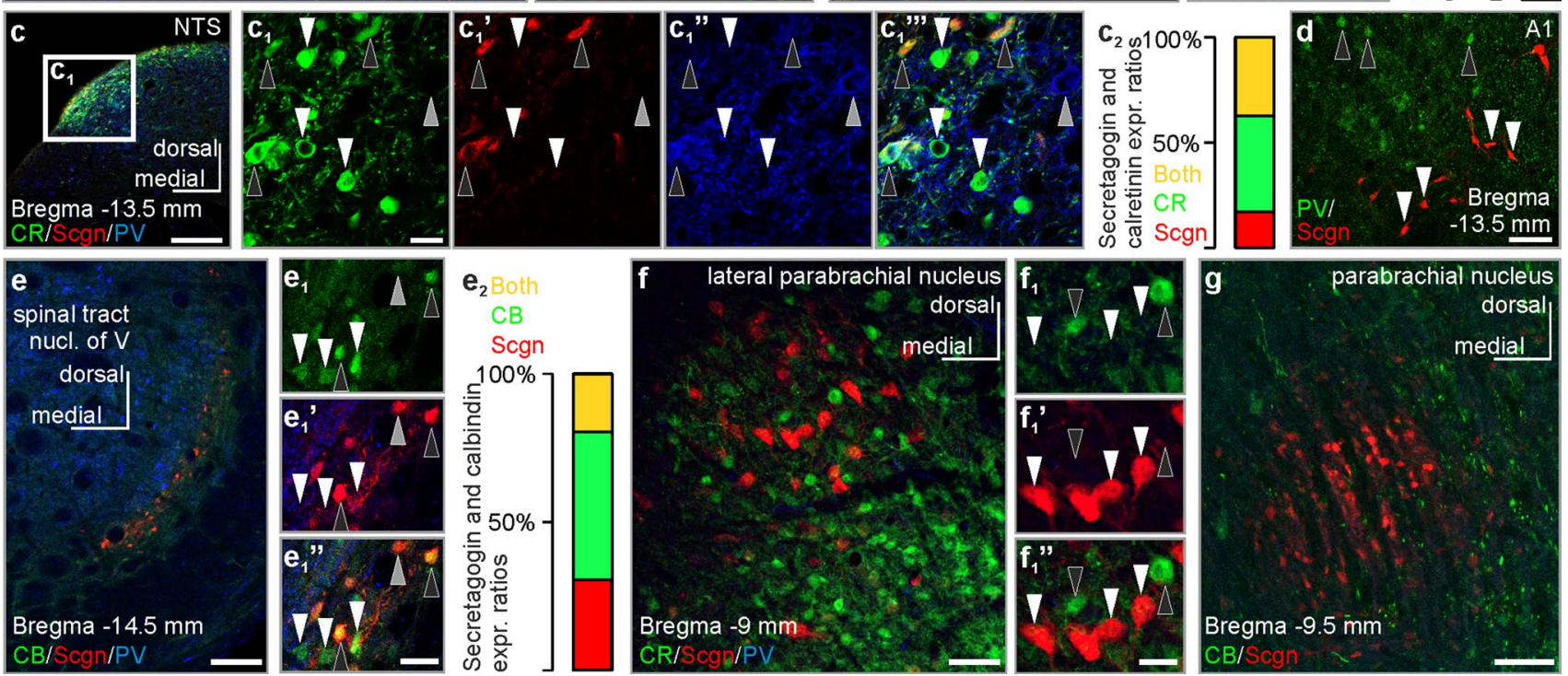

Fig. 5 Co-expression of secretagogin with classical $\mathrm{Ca}^{2+}$-binding proteins in select brainstem nuclei of the rat. $\mathbf{a}-\mathbf{a}_{1}$ " Complementary distribution of calretinin and secretagogin in the commissural part of the solitary nucleus and the dorsal nucleus of vagus (white arrowheads indicate secretagogin ${ }^{+} /$calretinin $^{-}$neurons, black arrowhead points to a secretagogin ${ }^{+} /$calretinin $^{+}$neuron). $\mathbf{b}-\mathbf{b}_{1}{ }^{\text {' Secretagogin }}{ }^{+}$ neurons typically remained calretinin immunonegative (white arrowheads) in the spinal tract nucleus of the trigeminus, with exceptional co-expression only (black arrowhead). $\mathbf{b}_{2}$ Single- and co-expression ratios of secretagogin and calretinin in the spinal tract nucleus of the trigeminus. $100 \%$ percentage refers to all labelled cells detected for secretagogin and/or calretinin immunoreactivity. Secretagogin ${ }^{+} /$ calretinin $^{+}$neurons: $2.8 \pm 3.2 \%$, secretagogin ${ }^{+} /$calretinin $^{-}$neurons $51.2 \pm 6.1 \%$, secretagogin ${ }^{-} /$calretinin $^{+}$neurons $47.5 \pm 5.4 \%$ (as of average \pm s.e.m.). c- $\mathbf{c}_{\mathbf{1}}$ " " Calretinin ${ }^{+}$neurons in the dorsolateral part of the solitary tract nucleus can either remain immunonegative for secretagogin (white arrowheads) or co-express it (black arrowheads) Grey arrowhead points to a $\mathrm{CR}^{-} / \mathrm{Scgn}^{-} / \mathrm{PV}^{+}$neuron. $\mathbf{c}_{2}$ Single- and co-expression ratios of secretagogin and calretinin in the dorsolateral part of the solitary tract nucleus. $100 \%$ percentage refers to all labelled cells detected for secretagogin and/or calretinin immunoreactivity. Secretagogin ${ }^{+} /$calretinin $^{+}$neurons: $36.1 \pm 2.3 \%$, secretagogin ${ }^{+} /$ calretinin $^{-}$neurons $16.9 \pm 3.5 \%$, secretagogin ${ }^{-} /$calretinin $^{+}$neurons
$43.7 \pm 2.1 \%$ (as of average \pm s.e.m.). d Secretagogin ${ }^{+}$neurons (white arrowheads) in the A1 field did not co-express parvalbumin (black arrowheads point to secretagogin ${ }^{-} /$parvalbumin $^{+}$somata). e- $\mathbf{e}_{\mathbf{1}}$ ", Secretagogin ${ }^{+}$neurons typically co-expressed calbindin in the spinal tract nucleus of the trigeminus (black arrowheads). White arrowheads point to calbindin ${ }^{+} /$secretagogin $^{-}$somata, grey arrowhead to a secretagogin ${ }^{+} /$calbindin $^{-}$soma. $\mathbf{e}_{2}$ Single- and co-expression ratios of secretagogin and calbindin in the spinal tract nucleus of the trigeminus. $100 \%$ percentage refers to all labelled cells detected for secretagogin and/or calbindin immunoreactivity. Secretagogin ${ }^{+} /$ calbindin $^{+}$neurons: $19.1 \pm 8.0 \%$, secretagogin ${ }^{+} /$calbindin $^{-}$neurons $30.7 \pm 11.3 \%$, secretagogin ${ }^{-} /$calbindin $^{+}$neurons $50.2 \pm 7.2 \%$ (as of average \pm s.e.m.). $\mathbf{f}-\mathbf{f}_{\mathbf{1}}{ }^{\prime}$ 'Secretagogin ${ }^{+}$neurons showed a complementary distribution to calretinin ${ }^{+}$neurons in the lateral parabrachial nucleus (white arrowheads point to secretagogin ${ }^{+} /$calretinin $^{-}$somata, black arrowheads point to secretagogin $^{-} /$calretinin $^{+}$somata). $\mathbf{g}$ Secretagogin ${ }^{+}$neurons remain immunonegative for calbindin in the parabrachial nucleus. $C B$ calbindin, $C R$ calretinin, $N T S$ solitary tract nucleus, NTSc commissural part of the solitary tract nucleus, Scgn secretagogin, $P V$ parvalbumin, $X$ dorsal nucleus of vagus, $X I I$ hypoglossal nucleus. Scale bars $150 \mu \mathrm{m}(\mathbf{a}, \mathbf{b}, \mathbf{c}, \mathbf{e}), 70 \mu \mathrm{m}(\mathbf{g}), 40 \mu \mathrm{m}(\mathbf{d}$, f), $10 \mu \mathrm{m}\left(\mathbf{a}_{\mathbf{1}}{ }^{\prime}, \mathbf{b}_{\mathbf{1}}, \mathbf{c}_{\mathbf{1}}, \mathbf{e}_{\mathbf{1}}, \mathbf{f}_{\mathbf{1}},{ }^{\prime}\right)$ 

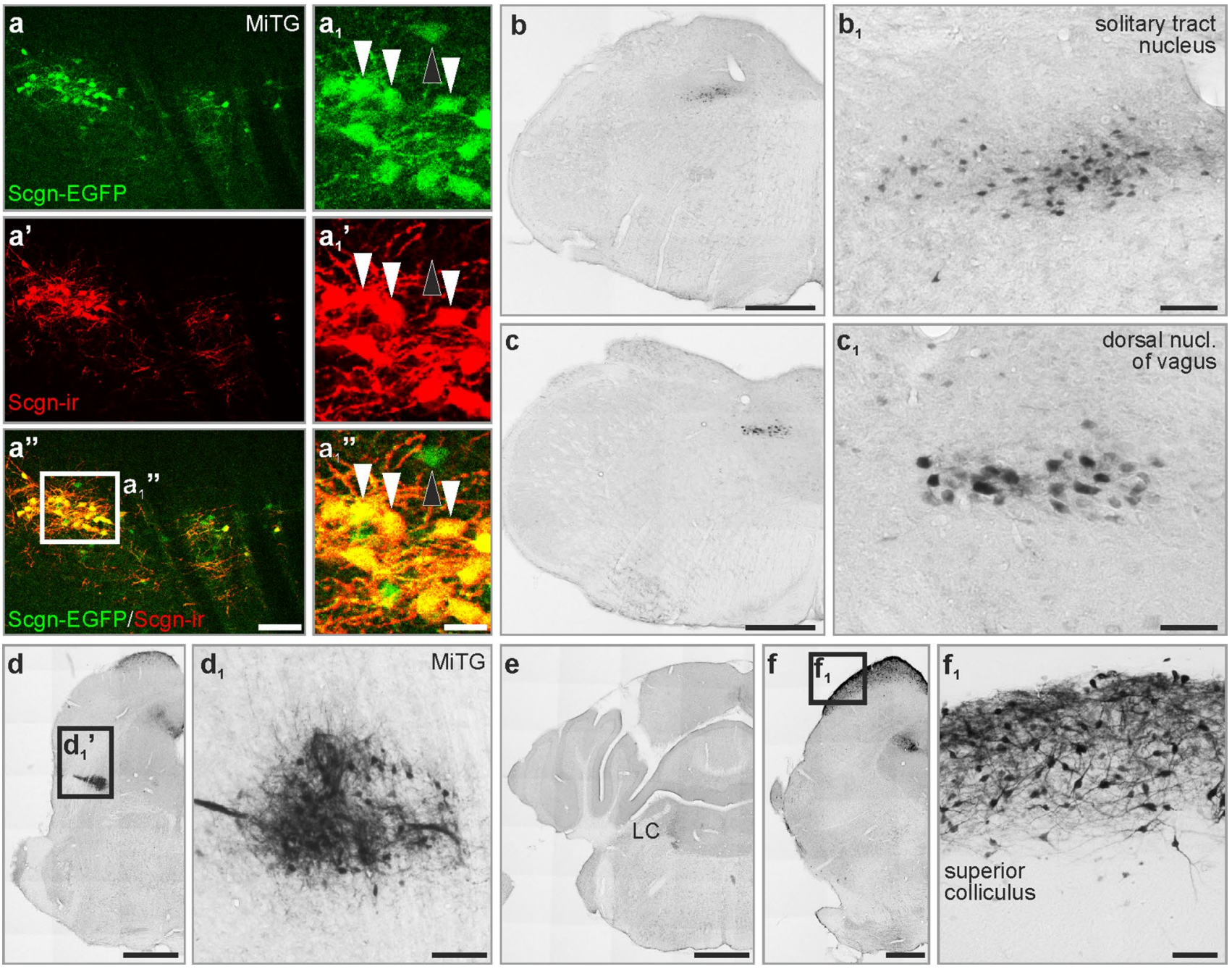

Fig. 6 Secretagogin expression in the mouse brain stem. $\mathbf{a}-\mathbf{a}_{1}$," EGFP-expressing neurons in secretagogin-EGFP mice showed secretagogin immunoreactivity as exemplified in the microcellular tegmental nucleus (white arrowheads in $\mathbf{a}-\mathbf{a}_{1}$ "). Occasionally, EGFPexpressing neurons remained secretagogin immunonegative (black arrowhead in $\mathbf{a}-\mathbf{a}_{\mathbf{1}}$ "'). $\mathbf{b}-\mathbf{f}_{\mathbf{1}}$ Secretagogin ${ }^{+}$neurons in the solitary tract nucleus $\left(\mathbf{b}, \mathbf{b}_{\mathbf{1}}\right)$, dorsal nucleus of vagus $\left(\mathbf{c}, \mathbf{c}_{\mathbf{1}}\right)$, microcellular tegmental nucleus $\left(\mathbf{d}, \mathbf{d}_{\mathbf{1}}\right)$, locus coeruleus (e) and the superior colliculus (f, $\mathbf{f}_{1}$ ). $L C$ locus coeruleus, $M i T g$ microcellular tegmental nucleus. Scale bars $1 \mathrm{~mm}(\mathbf{b}-\mathbf{f}), 100 \mu \mathrm{m}\left(\mathbf{a}\right.$ ", $\left.\mathbf{d}_{\mathbf{1}}, \mathbf{f}_{\mathbf{1}}\right), 70 \mu \mathrm{m}\left(\mathbf{b}_{\mathbf{1}}\right), 40 \mu \mathrm{m}\left(\mathbf{c}_{\mathbf{1}}\right)$

\section{Secretagogin expression is reduced in noradrenergic brainstem nuclei in Alzheimer's disease}

Immersion fixation of blocked tissue, the routine processing procedure, and post-mortem delay adversely impact epitope detection by immunohistochemistry in human brain tissue. We overcame this problem by applying vascular perfusion via the internal carotid and vertebral arteries to preserve tissue integrity. Similarly to laboratory rodents, we found secretagogin ${ }^{+}$neurons in the noradrenergic axis of the human brainstem (Fig. 8a-c), including the locus coeruleus (Fig. 8b, $b_{1}$ ), and A1 (Fig. 8a $a_{2}$ ) and A7 (Fig. 8c) nuclei. $\mathrm{TH}^{+}$locus coeruleus neurons co-expressed secretagogin, with subcellular foci (patches) restricted to peripheral intrasomatic domains and neuronal processes, frequently apposing the cell membrane (Fig. 8d-d ${ }_{1}$ '). In addition, we identified secretagogin ${ }^{+}$ neurons in the dorsal nucleus of vagus of the human brain (Fig. 8a, $\mathrm{a}_{1}$ ), similarly to what we found in rat (Fig. $4 \mathrm{~d}_{1}{ }^{\text {') }}$ and mouse (Fig. 6c $\mathrm{c}_{1}$ ).

We hypothesized that neurodegenerative stimuli may be harmful for secretagogin expression in LC neurons. Alternatively, secretagogin could spare these cells from neurodegeneration. Secretagogin ${ }^{+}$hippocampal or olfactory neurons are preserved in neurodegenerative disorders, like Alzheimer's disease (Attems et al. 2008, 2012a), supporting the latter hypothesis. However, it has 

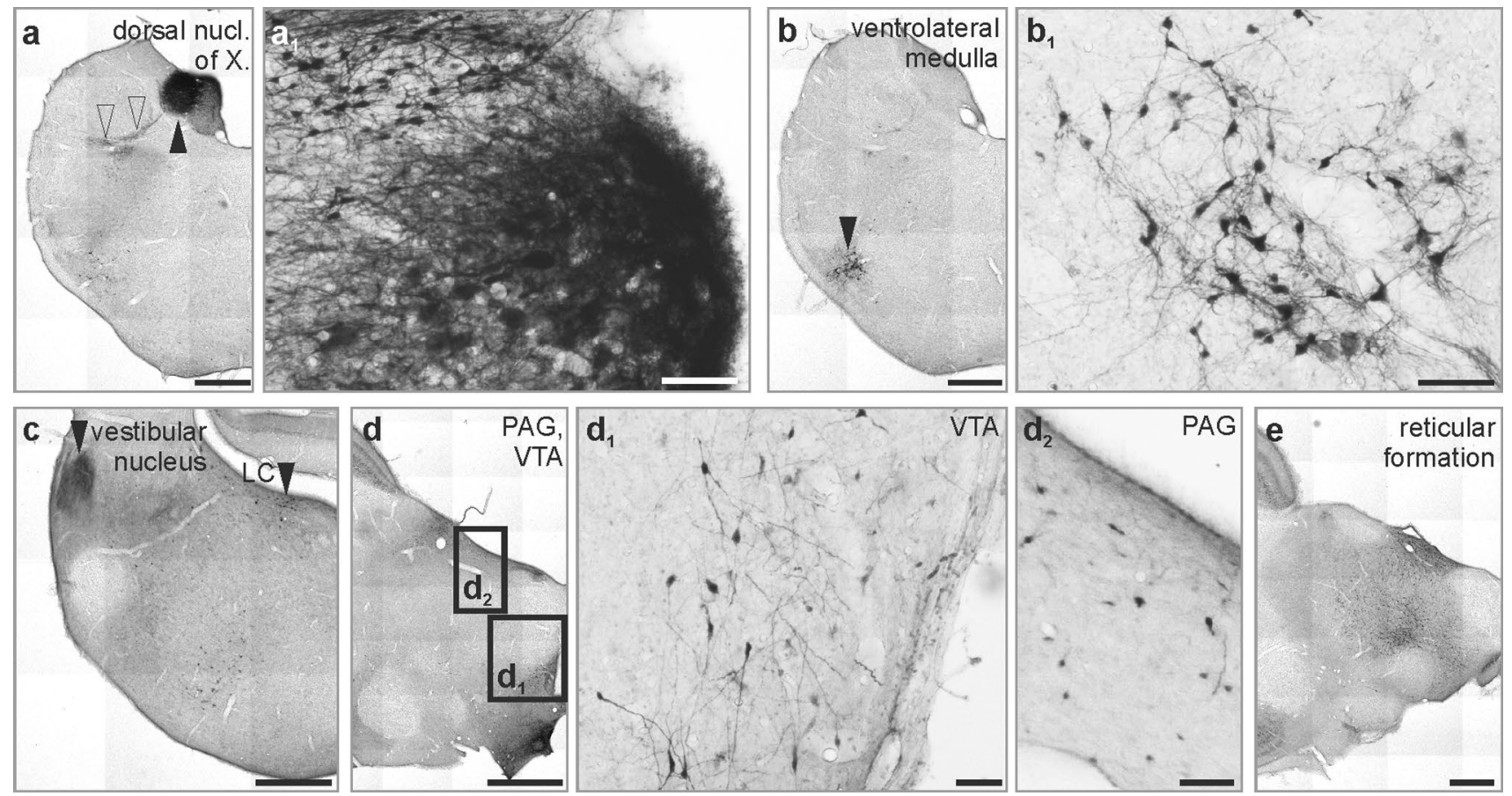

Fig. 7 Secretagogin expression in the chicken brain stem. a-e The distribution of secretagogin ${ }^{+}$neurons in the avian brain was similar to what we found in the mammalian brain. $\mathbf{a}-\mathbf{b}_{\mathbf{1}}$ In the medulla oblongata, immunoreactive neurons populated the dorsal nucleus of vagus (black arrowhead in a, open arrowheads indicate nerve fibres emanating from the nucleus) and the ventrolateral medulla $\left(\mathbf{b}, \mathbf{b}_{1}\right)$. $\mathbf{c}$ In the pons, immunoreactivity was typically confined to the vestibular area and the locus coeruleus. $\mathbf{d}-\mathbf{e}$ In the midbrain, neurons of the periaqueductal grey matter, of the ventral tegmental area and scattered cells in the reticular formation showed secretagogin immunoreactivity. $L C$ locus coeruleus, $P A G$ periaqueductal grey, VTA ventral tegmental area. Scale bars $1 \mathrm{~mm}(\mathbf{a}-\mathbf{e}), 50 \mu \mathrm{m}\left(\mathbf{a}_{1}, \mathbf{b}_{1}, \mathbf{d}_{1}, \mathbf{d}_{2}\right)$ been proposed that $\mathrm{TH}^{+}$locus coeruleus neurons are most sensitive and compromised already in the early phase in Alzheimer's disease (Tomlinson et al. 1981): tau pathology appears earliest in locus coeruleus noradrenaline neurons (Attems et al. 2012b). Monitoring secretagogin expression at the transcription (RNA) level, we found that it robustly decreased already in Braak III-IV stage subjects and further diminished in severe Alzheimer's disease (Fig. 8h). In severe Alzheimer's disease subjects (Braak $\mathrm{V}-\mathrm{VI}$ ), we identified a parallel decrease in TH (relative fluorescence intensity values: $103.59 \pm 5.82$ in Braak I-II vs. $52.66 \pm 1.55$ in Braak V-VI) and secretagogin (relative fluorescence intensity values: $47.13 \pm 0.03$ in Braak I-II vs. $27.42 \pm 1.06$ in Braak V-VI) immunoreactivity (Fig. 8e-g'), In individuals with advanced stage of Alzheimer's disease-related pathology (Braak V-VI), neurons with abundant cytoplasmic secretagogin immunoreactivity were very rare and often lacked AT8-immunoreactivity (hyperphosphorylated tau) (Fig. 8i, i'). Typically, secretagogin expression within $\mathrm{TH}^{+}$neurons, if detectable, was restricted to very small intracellular compartments. These foci often overlapped with the $\mathrm{AT} 8^{+}$microdomains where tau hyperphosphorylation started to develop within the neuron (Fig. $8 \mathrm{f}_{1}-\mathrm{f}_{1}$ ', , but see also Fig. $8 \mathrm{f}_{2}-\mathrm{f}_{2}{ }^{\prime}$ '). Secretagogin expression was more evident in neuronal processes which occasionally showed multiple islets of $\mathrm{AT}^{+}$profiles (Fig. 8j-j'”). In conclusion, secretagogin expression decreased with the progression of Alzheimer's disease, with residual immunoreactivity in degenerating intracellular microdomains.

\section{Discussion}

The present study underlines the usefulness of secretagogin as a novel neurochemical marker to distinguish subsets of neurons in the vertebrate brain. Detected already during early ontogeny, secretagogin in adults was then expressed in important relay centres in the lower brain stem, notably in several of the noradrenaline groups, with the A6 locus coeruleus being of particular interest. Moreover, secretagogin expression in the locus coeruleus from Alzheimer's disease subjects paralleled TH loss and was associated with initial, aberrant tau phosphorylation. 


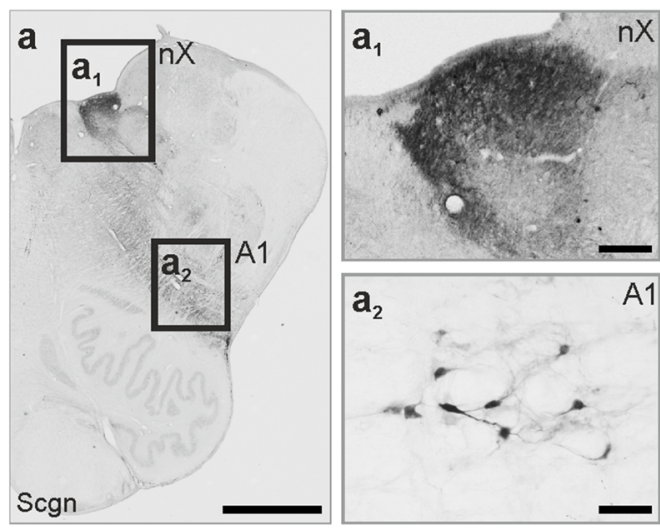

Braak I-II
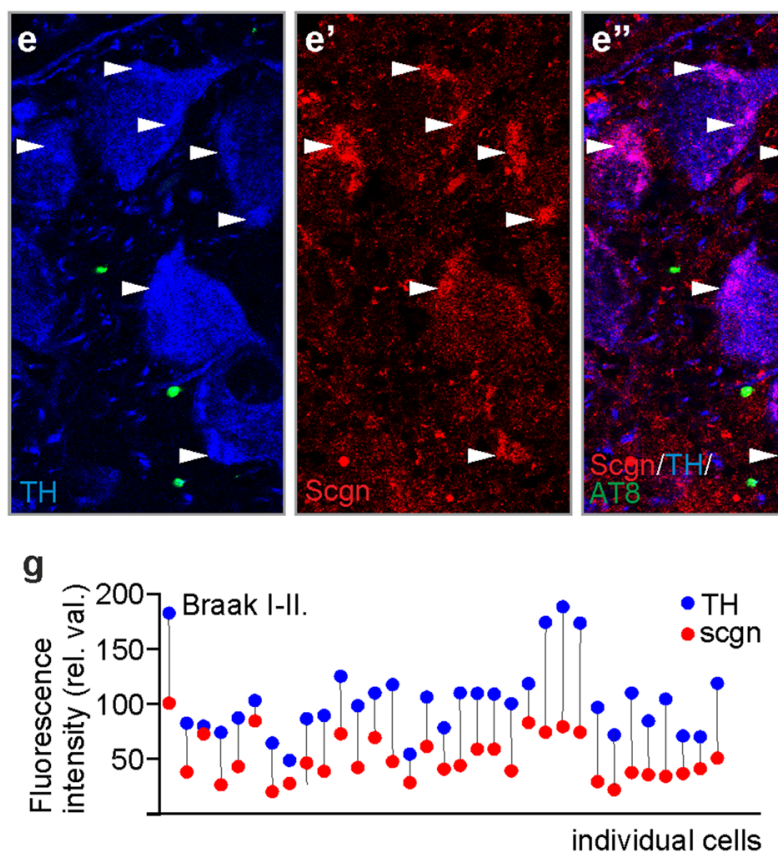
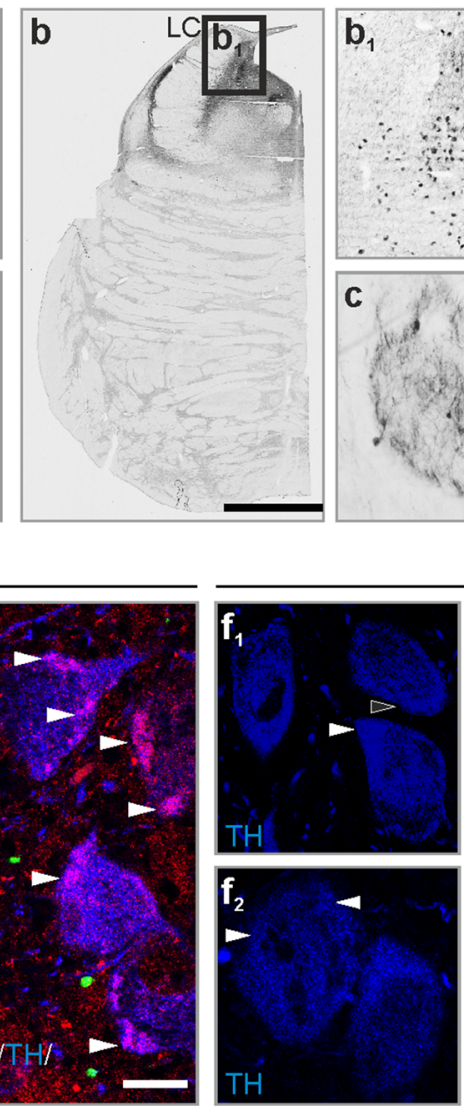

g'

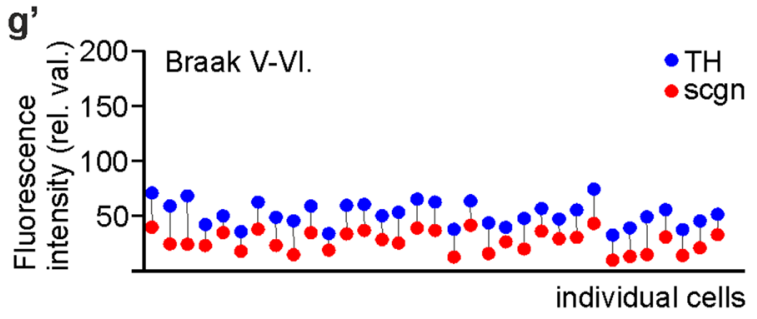

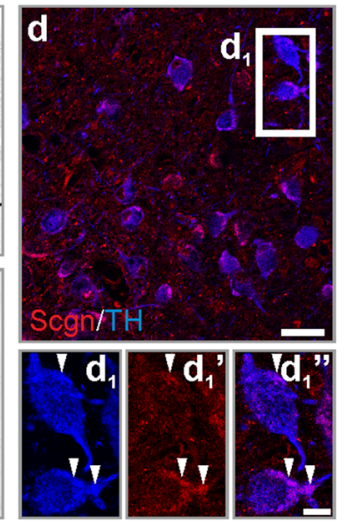

Braak V-VI

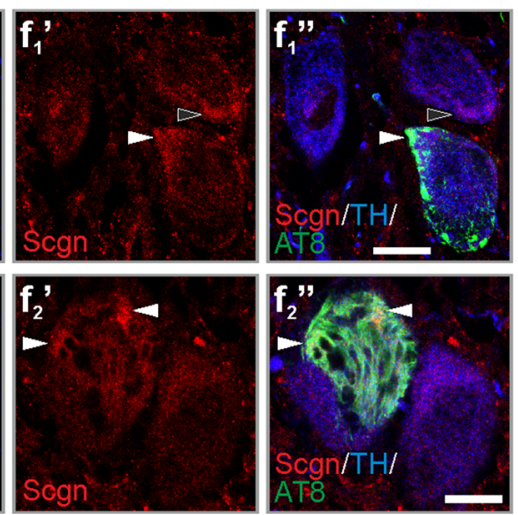

$\mathrm{TH}$

Braak V-VI
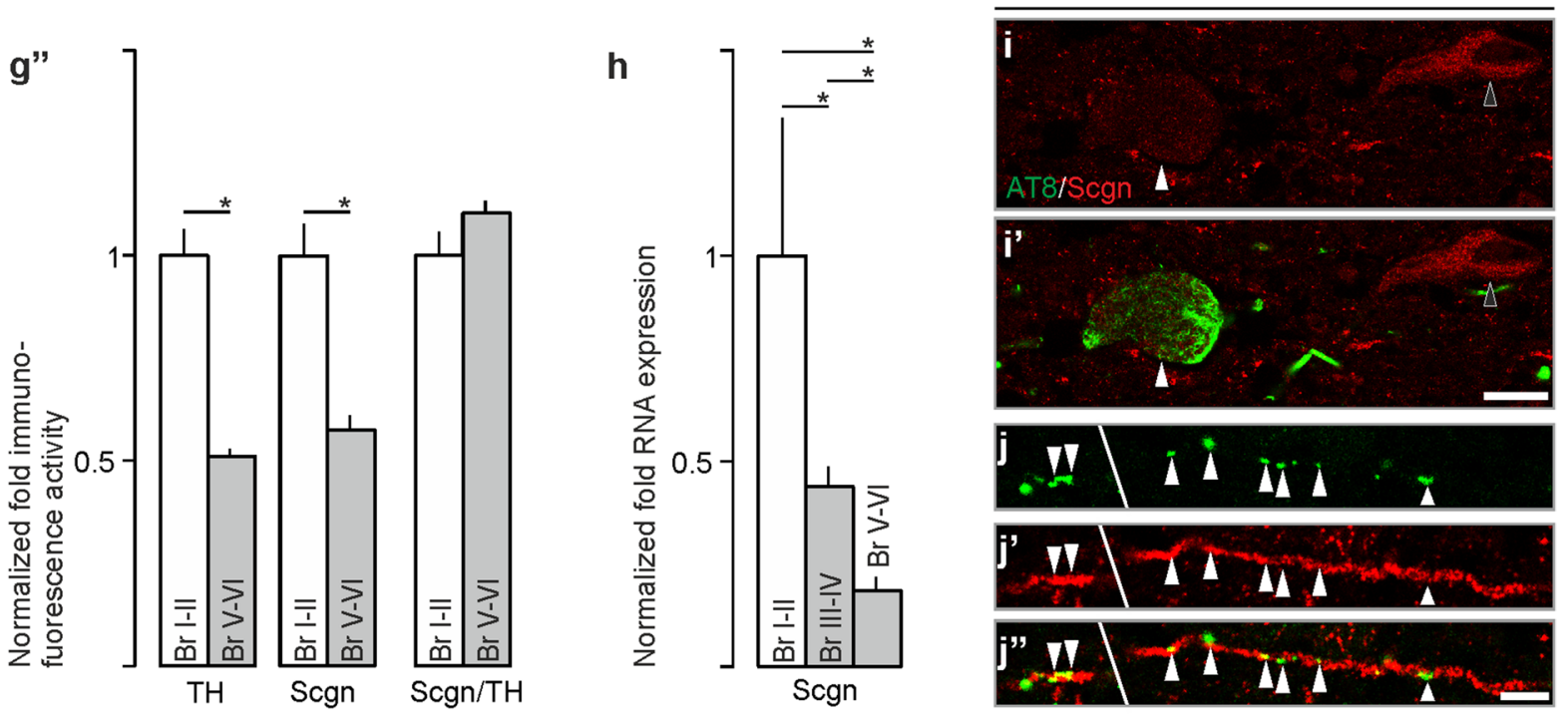

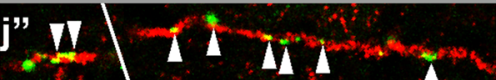


4Fig. 8 Secretagogin expression in the human brainstem is compromised in Alzheimer's disease. $\mathbf{a}-\mathbf{a}_{2}$ Secretagogin $^{+}$neurons of the medulla oblongate populated the dorsal nucleus of vagus $\left(\mathbf{a}_{1}\right)$, captured from the immediate consecutive section after the section shown in $\mathbf{a}$ and the A1 field $\left(\mathbf{a}_{2}\right)$. b, $\mathbf{b}_{1}$ In the pons, secretagogin ${ }^{+}$neurons typically occurred in the locus coeruleus. c Secretagogin ${ }^{+}$neurons in the A7 field. $\mathbf{d}-\mathbf{d}_{\mathbf{1}}$ " $\mathrm{TH}^{+}$neurons of the human locus coeruleus expressed secretagogin. Secretagogin immunoreactivity concentrated along the cell membrane and most typically where dendrites emanated from the soma (arrowheads in $\mathbf{d}-\mathbf{d}_{\mathbf{1}}$ "). $\mathbf{e}-\mathbf{e}$ " Secretagogin was abundantly expressed in $\mathrm{TH}^{+}$locus coeruleus neurons in individuals without histopathological signs of Alzheimer's disease (Braak I-II) (arrowheads in e-e" point to secretagogin-immunoreactive loci in $\mathrm{TH}^{+}$soma. $\mathbf{f}_{\mathbf{1}}-\mathbf{f}_{\mathbf{2}}$ ', In subjects with severe Alzheimer's disease (Braak $\mathrm{V}-\mathrm{VI})$, secretagogin ${ }^{+}$subcellular domains in $\mathrm{TH}^{+}$somata were significantly reduced and often overlapped with the accumulation foci of $\mathrm{AT}^{+}$hyperphosphorylated tau (arrowheads in $\mathbf{f}_{\mathbf{1}}-\mathbf{f}_{\mathbf{2}}$ ', , black arrowhead in $\mathbf{f}_{\mathbf{1}}-\mathbf{f}_{\mathbf{1}}$, points to a secretagogin ${ }^{+} / \mathrm{AT}^{-}{ }^{-}$domain). $\mathbf{g}, \mathbf{g}^{\prime} \mathrm{TH}$ and secretagogin immunofluorescence showed a paralleled reduction in individual locus coeruleus neurons in Braak V-VI vs. Braak I-II subjects. g' In average, TH-immunoreactivity decreased in locus coeruleus somata in severe Alzheimer's disease which was paralleled with a loss in secretagogin immunoreactivity. $\mathbf{h}$ Secretagogin mRNA expression decreased significantly with the progress of Alzheimer's disease in locus coeruleus micropunches. $\mathbf{i}-\mathbf{i}$ Complementary distribution of AT8 and secretagogin immunoreactivity of locus coeruleus neurons in severe Alzheimer's disease. $\mathbf{j}-\mathbf{j}$ ' Initial, discontinuous accumulation of $\mathrm{AT}^{+}$tau protein (arrowheads) in secretagogin ${ }^{+}$neuronal process in the locus coeruleus of a late-stage Alzheimer's disease subject. $\mathbf{e}_{\mathbf{1}}, \mathbf{f}_{\mathbf{1}}, \mathbf{h} p<0.05$, Student's $t$ test. Scgn secretagogin, TH tyrosine hydroxylase. Scale bars $500 \mu \mathrm{m}(\mathbf{a}, \mathbf{b}), 100 \mu \mathrm{m}\left(\mathbf{a}_{\mathbf{1}}, \mathbf{b}_{\mathbf{1}}, \mathbf{c}\right)$, $25 \mu \mathrm{m}\left(\mathbf{a}_{2}\right), 15 \mu \mathrm{m}(\mathbf{d}), 5 \mu \mathrm{m}\left(\mathbf{d}_{\mathbf{1}}\right.$ ',, $\mathbf{e}-\mathbf{e}$ ', $, \mathbf{i}-\mathbf{i} ', \mathbf{f}_{\mathbf{1}}-\mathbf{f}_{\mathbf{2}}$ '), $500 \mu \mathrm{m}(\mathbf{a}, \mathbf{b})$, $3 \mu \mathrm{m}$ (j'). Images $\mathbf{a}, \mathbf{b}, \mathbf{c}$ and $\mathbf{d}-\mathbf{d}_{\mathbf{1}}$ ', were reproduced with permission from EMBO: Alpár et al., Hypothalamic CNTF volume transmission shapes cortical noradrenergic excitability upon acute stress. EMBO J. 2018 Nov 2;37(21). pii: e100087

\section{Secretagogin is expressed early during ontogenesis and persists throughout adulthood in locus coeruleus neurons}

CaBPs appear at different time points and last for different intervals during brainstem development. Calretinin, calbindin and parvalbumin are sequentially expressed in the rat auditory brainstem during development, correlating with definite developmental stages (Lohmann and Friauf 1996). The postnatal development of rat vestibular nuclei and their cerebellar projections could be mapped by the specific spatiotemporal appearance of the same CaBPs (Puyal et al. 2002). Notably, the early onset of the expression of these $\mathrm{CaBPs}$ is no guarantee for their ongoing presence through adulthood. For example, even if most neurons, which express calbindin D28k during development preserve their phenotype (Enderlin et al. 1987), this is not the case in the superior olivary complex, where calbindin D28k expression is transient and may reflect critical periods in the control of calcium homoeostasis (Friauf 1993). Calretinin's transient expression during ontogenesis is even more pronounced, in mammals (Lohmann and Friauf 1996), fish (Porteros et al.
1998) and avian species (Bastianelli and Pochet 1993). Parvalbumin, in turn, may appear late (in fact postnatal) during forebrain development (Lohmann and Friauf 1996) and is induced by specific afferent stimuli (Barker and Dreher 1998; Manns and Gunturkun 2003; McHaffie et al. 2001). We here show that secretagogin appears early in mouse brainstem development and shows ongoing expression in mouse brainstem noradrenergic cells, notably of the locus coeruleus neurons. Speculatively, secretagogin may regulate calcium-dependent mechanisms or TH synthesis in noradrenergic neurons, which are critical not only in adulthood but already in embryonic life.

\section{Secretagogin expression in relay, vegetative and stress centres of the vertebrate brainstem}

Secretagogin was expressed in nuclei with different functions. We highlight that secretagogin ${ }^{+}$neurons (i) populate the brainstem nuclei which serve as major vegetative command centres, such as the parabrachial or the solitary tract nucleus, (ii) occur in critical relay stations in the pathway of special senses (vestibular and visual), (iii) outline the brainstem noradrenaline axis and (iv) that this expression pattern has been preserved in phylogenesis, from avian species to humans. At the same time, secretagogin was expressed neither in serotonin ${ }^{+}$raphe neurons nor in $\mathrm{TH}^{+}$neurons in the ventral tegmentum, i.e. dopamine neurons, and we could reconfirm (data not shown) that cholinergic neurons at the midbrain-hindbrain border, including the pedunculopontine nucleus (Ch5), the laterodorsal tegmental nucleus (Ch6) and the parabigeminal nucleus (Ch8), remained immunonegative for secretagogin (Kosaka and Kosaka 2018).

$\mathrm{CaBPs}$ are widely used as neurochemical markers to distinguish neuronal pools (Andressen et al. 1993; Celio 1990; Jacobowitz and Winsky 1991), with their exact intracellular function, however, remaining contradictory (Freund et al. 1990) or largely unrevealed. In general terms, $\mathrm{Ca}^{2+}$-sensor proteins, including secretagogin (Wagner et al. 2000), undergo a conformational change upon cell activation (Rogstam et al. 2007) to trigger intracellular signalling events (Gartner et al. 2007; Hanics et al. 2017; Malenczyk et al. 2017). Thus, secretagogin can be critical to regulate vegetative functions and sensory processing at the brainstem level based on their specific expression in the above nuclei. To unravel secretagogin's physiological role in brainstem nuclei, future experiments could investigate the vegetative, vestibular and visual systems. We would definitely consider loss-of-function and activity-dependence experiments which impact the vegetative control at the major supraspinal level (solitary tract nucleus), control of balance and relevant motor skills as well as visual functions controlled at the midbrain level (e.g. smooth pursuit). Finally, whilst we recently explored secretagogin's role in a mechanism converting 
hypothalamic activation into long-lasting cortical excitability following acute stress (Alpar et al. 2018), secretagogin's function in shaping the ascending and descending reticuloactivating system is a promising field for future works.

\section{Is secretagogin involved in somato-dendritic release of neuropeptides?}

It has been shown that secretagogin interacts with proteins implicated in i.a. docking of release vesicles (Bauer et al. 2011; Romanov et al. 2015). In fact, there is evidence that secretagogin is localized in corticotropin-releasing hormone (CRH) axon terminals at the median eminence and modulates release of this neuropeptide at this site (Romanov et al. 2015). The present results show that secretagogin in human locus coeruleus neuron cell bodies and dendrites has a patchy distribution not rarely close to the cell membrane. We have proposed that the neuropeptide galanin is released from soma and dendrites of rat (Vila-Porcile et al. 2009) and human (Barde et al. 2016) locus coeruleus neurons under stressful conditions. This type of release has been shown for oxytocin and vasopressin to occur in the hypothalamic magnocellular neurons (Ludwig and Leng 2006). It may be relevant to analyse if secretagogin in the locus coeruleus neurons is involved in the regulation of galanin release, since this peptide system is involved in mood disorders (Hokfelt et al. 2018; Holmes and Picciotto 2006; Kuteeva et al. 2010; Lu et al. 2007).

\section{Secretagogin expression decreases in Alzheimer's disease}

$\mathrm{Ca}^{2+}$-sensor proteins work in an activity-dependent manner to control downstream signalling. Cell activity and excitability are reduced in neurodegenerative diseases, including Alzheimer's disease (de Haan et al. 2017). We found that secretagogin expression significantly decreased with disease progression, paralleling TH loss typical already in the early phase of Alzheimer's disease (Braak and Del Tredici 2012; Chan-Palay and Asan 1989; German et al. 1992; Kelly et al. 2017), which may reflect impaired cell function and activity.

Previous studies suggested that secretagogin is neuroprotective in hippocampal pyramidal cells in Alzheimer's disease (Attems et al. 2008) and secretagogin expression has recently been shown to parallel disease progression: P301L tau transgenic mice showed reduced secretagogin expression in hippocampal neurons (Attems et al. 2011). Whilst we found that structural malformations can be independent of secretagogin loss (neurons with robustly reduced or not detectable secretagogin expression did not necessarily contain $\mathrm{AT}^{+}{ }^{+}$hyperphosphorylated tau), small intracellular domains with residual secretagogin expression were identified as foci with initial tau aberrancies and islet-like multiple accumulation of $\mathrm{AT}^{+}$tau repeatedly occurred in the secretagogin ${ }^{+}$processes. This may implicate that secretagogin-expressing domains can resist structural degradation which refers to its previously suggested role in neuroprotection (Attems et al. 2008). Accumulating evidence suggest that a deregulation of calcium signalling may play a major role in Alzheimer's disease progression; CaBPS such as parvalbumin, calbindin and calretinin are upregulated in the hippocampus of 3-month-old APPswe/ PS1dE9 transgenic mice, possibly to control cellular homoeostasis and synaptic plasticity, but losing cellular capacity to pathophysiological processes by the age of 12 months (Verdaguer et al. 2015). Activity-dependent translocation of synaptonuclear factors from synapses to the nucleus is regulated by calmodulin-dependent mechanisms; altered synapse-to-nucleus signalling may lead to neurodegenerative and neuropsychiatric diseases (Parra-Damas and Saura 2019). $\mathrm{Ca}^{2+} /$ calmodulin-dependent protein kinase kinase 2 controls important neuronal processes and its loss leads to aberrant transferrin phosphorylation and trafficking which makes it a potential biomarker for Alzheimer's disease (Sabbir 2018). CaBPs-mediated mechanisms are also important in glial cells to resist neurological disorders. Calcium dysregulation triggers astrocyte activation which leads to neuroinflammation, release of synaptotoxic factors and loss of glutamate regulation which can finally culminate in neurodegeneration (Sompol and Norris 2018). Dysregulation of calcineurin signalling pathways in activated astrocytes and its interaction with the nuclear factor of activated $T$ cells (NFATs) couple vascular pathology to neurodegeneration and cognitive loss (Kraner and Norris 2018). Calcium sensor proteins have been previously implicated in neuroprotection: dopamine neurons in the substantia nigra use calretinin to confer oestrogen's effect to prevent cell loss (Yi et al. 2016) and calretinin cooperates with the NCX1 exchanger to resist neurodegeneration (Boscia et al. 2016).

We suggest that secretagogin's involvement in Alzheimer's disease is (i) due to its calcium sensor rather than to its calcium buffer property. Secretagogin has been shown to regulate diverse cellular mechanisms and functions, including migration or hormonal release, in distinct brain neuronal populations (Alpar et al. 2018; Hanics et al. 2017; Romanov et al. 2015). All these functions seemed to be linked to secretagogin's calcium sensor property. Second (ii), secretagogin's involvement in Alzheimer's disease is likely exerted by affecting noradrenergic cell function, hence modulating noradrenergic cortical input. In conclusion, secretagogin as a calcium sensor may act neuroprotectively by regulating downstream machineries in noradrenergic cells. 


\section{Materials and methods}

\section{Animals, surgery and ethical approval of experimental studies}

Eleven 6-week-old male rats (Wistar), three 12-week-old male wild-type mice (C57BL/6), a total of six embryos from three pregnant C57BL/6 mice, three 12-week-old male EGFP-secretagogin transgenic mice and three 14-day-old chicks (Gallus domesticus) were used. Food and water were available ad libitum and animals were kept under standard housing condition and using a 12/12 light/dark cycle. Experimental procedures, including stereotaxic injections and transcardial perfusion, were approved by the Ethical Review Board of the Semmelweis University and conformed to the European Convention for the Protection of Vertebrate Animals used for experimental and other scientific purposes (Protocols: ETS No. 170, ETS No.123, Tierversuchgesetz 2012, BGBI, Nr. 114/2012). Animals during surgeries and transcardial perfusions were anesthetized intramuscularly (i.m.) or intraperitoneally (i.p.) with a mixture of ketamine $(50 \mathrm{mg} / \mathrm{kg} \mathrm{b} \mathrm{wt})$ and xylazine (4 mg/kg b wt). After surgery, brains were perfusion fixed transcardially with $4 \%$ paraformaldehyde (PFA) in $0.1 \mathrm{M}$ phosphate buffer $(0.1 \mathrm{M} \mathrm{PB})$.

\section{Production of the SB-BAC-SCGN-EGFP transgenic mouse line}

To generate transgenic mice expressing the EGFP (enhanced green fluorescent protein) under the control of the SCGN promoter, we used a BAC (Bacterial Artificial Chromosome) engineering technology (Lee et al. 2001). The B6Ng01268C03 (RIKEN BioResource Research Center) BAC clone was chosen, which contained the whole SCGN gene and downstream of it another harbouring gene (Hist1h2ba) which was removed later. In the BAC modification casette, the EGFP cDNA was inserted between the recombination arms and its ATG site was fused into the SCGN's gene ATG site. Following the EGFP cDNA, a WPRE (woodchuck hepatitis virus (WHP) posttranscriptional regulatory element), an hGH-PA (human growth hormone polyadenylation signal) and a neomycin selection marker flanked by flippase recognition sites were inserted into the construct. Recombination was carried out as described by Lee et al. (2001). After the insertion of the EGFP and other components into the BAC, the neomycin marker was removed in the host cells by a flippase enzyme. This was followed by a second recombineering-based BAC modification step in which $40 \mathrm{~kb}$ downstream region from the SCGN gene was removed, which contained the neighbour gene. In the same step, we inserted both ITR (inverted terminal repeat) sequences recognized by the Sleeping Beauty transposase to combine the BAC transgenesis with a transposon-based system for increased integration efficiency (Rostovskaya et al. 2012). Transgenic mice were derived by pronuclear microinjection of the SB-BAC-SCGN-EGFP BAC (circular, $1 \mathrm{ng} / \mu \mathrm{l})$, Sleeping Beauty transposase mRNA $(5 \mathrm{ng} / \mu \mathrm{l})$ into C57BL/6Ntac fertilized eggs.

\section{Immunohistochemistry and imaging}

Chromogenic or multiple immunofluorescence histochemistry with select combinations of primary antibodies (Table 1) was performed according to published protocols (Alpar et al. 2010; Lendvai et al. 2013). Free-floating sections $(30 \mu \mathrm{m})$ were rinsed in phosphate buffer ( $\mathrm{PB}, \mathrm{pH} 7.4)$ and pre-treated with $0.3 \%$ Triton $\mathrm{X}-100$ (in PB) for $1 \mathrm{~h}$ at $22-24{ }^{\circ} \mathrm{C}$ to enhance the penetration of antibodies. Non-specific immunoreactivity was suppressed by incubating our specimens in a cocktail of 5\% normal donkey serum (NDS; Jackson), $2 \%$ bovine serum albumin (BSA; Sigma) and 0.3\% Triton X-100 (Sigma) in PB for $1 \mathrm{~h}$ at $22-24{ }^{\circ} \mathrm{C}$. Sections were exposed (16-72 $\mathrm{h}$ at $4{ }^{\circ} \mathrm{C}$ ) to select combinations of primary antibodies (Table 1, (Alpar et al. 2004; Hanics et al. 2017; Lendvai et al. 2013; Mulder et al. 2010; Romanov et al. 2015)) diluted in PB to which $0.1 \%$ NDS and $0.3 \%$ Triton X-100 had been added. After extensive rinsing in $\mathrm{PB}$, the sections
Table 1 List of markers used for immunolabelling

\begin{tabular}{lllll}
\hline Marker & Source & Host & IH dilution & References \\
\hline Calbindin & Synaptic systems & guinea pig, $\mathrm{pc}^{2}$ & $1: 1000$ & Hanics et al. (2017) \\
Calretinin & Synaptic systems & guinea pig, $\mathrm{pc}^{2}$ & $1: 1000$ & Hanics et al. (2017) \\
Parvalbumin & Millipore & Mouse, $\mathrm{mc}^{1}$ (AT8) & $1: 1000$ & Lendvai et al. (2013) \\
PHF tau & Pierce & Mouse, $\mathrm{mc}^{1}$ (AT8) & $1: 1000$ & Lendvai et al. (2013) \\
Secretagogin & L. Wagner & Rabbit, $\mathrm{pc}^{2}$ & $1: 12,000$ & Romanov et al. (2015) \\
Secretagogin & Cell signaling & Rabbit, $\mathrm{pc}^{2}$ & $1: 1000$ & This study \\
Secretagogin & R\&D systems & Goat, $\mathrm{pc}^{2}$ & $1: 100$ & Mulder et al. (2010) \\
Tyrosine hydroxylase & MERCK/Millipore & Rabbit, $\mathrm{pc}^{2}$ & $1: 1000$ & Alpar et al. (2004) \\
\hline
\end{tabular}

${ }^{1}$ Monoclonal antibody

${ }^{2}$ Polyclonal antibody 
were processed using chromogenic or immunofluorescence detection. In single-labelling experiments, the sections were exposed to biotinylated anti-rabbit IgG raised in donkey (1:1,000 [Jackson], $2 \mathrm{~h}$ at $22-24{ }^{\circ} \mathrm{C}$ ) followed by pre-formed avidin-biotin complexes also incorporating horseradish peroxidase for $1 \mathrm{~h}$ at $22-24^{\circ} \mathrm{C}$. Immunosignals were visualized by 3,3'-diaminobenzidine (Sigma, 0.025\%) as chromogen intensified with $\mathrm{Ni}$-ammonium sulphate $(0.05 \%$, Merck) in the presence of $0.001 \% \mathrm{H}_{2} \mathrm{O}_{2}$ as substrate (dissolved in $0.05 \mathrm{M}$ Tris buffer, $\mathrm{pH}$ 8.0). In multiple immunofluorescence labelling experiments, immunoreactivities were revealed by carbocyanine $(\mathrm{Cy})$ 2, 3 or 5-tagged secondary antibodies raised in donkey (1:200 [Jackson], $2 \mathrm{~h}$ at $\left.22-24{ }^{\circ} \mathrm{C}\right)$. Glassmounted sections were coverslipped with glycerol/gelatin (GG-1; Sigma).

The results of chromogenic stainings were captured on an Olympus BX-51 microscope at $10 \times, 20 \times$ and $40 \times$ primary magnification. Sections processed for multiple immunofluorescence histochemistry were inspected and images acquired on a 780LSM confocal laser-scanning microscope (Zeiss) with optical zoom ranging from $1 \times$ to $3 \times$ at $63 \times$ primary magnification (Plan-Apochromat $63 \times / 1.40$ ), and pinhole settings limiting signal detection to $0.5-0.7 \mu \mathrm{m}$ "optical thickness". Emission spectra for each dye were limited as follows: Cy2 (505-530 nm), Cy3 (560-610 nm), and Cy5 (650-720 nm). Multi-panel figures were assembled in CorelDraw X5 (Corel Corp.).

We used the histogram toolbox of the ZEN software (ZEISS) to measure immunofluorescence in the locus coeruleus neurons of Braak I-II $(n=3)$ and Braak V-VI $(n=3)$ subjects. Images from all sections $(n=3$ per subject) were captured using identical settings. Somata ( $n=11$ per section) were demarcated and their immunofluorescence, reflecting their TH and secretagogin immunoreactivity, were automatically measured and their quotient calculated.

To measure the overlap of secretagogin-EGFP expression and secretagogin immunoreactivity, we developed secretagogin immunoreactivity using the anti-secretagogin antibody raised in rabbits on sections made from secretagogin-EGFP mice brainstem. Analyses were carried out on images captured at $20 \times$ primary magnification. EGFP-expressing and immunoreactive somata were labelled with different markers in different layers (channels) using Photoshop which allowed co-localization of the two markers. In all three animals and for every nucleus, EGFP expression/immunoreactivity of somata were identified and marked in a minimum of three corresponding sections which were consecutive serial $30 \mu \mathrm{m}$-thick-sections of a four series-section pool. Labelled cells were counted in the whole nucleus on the section. The occurrence of secretagogin-EGFP expression or secretagogin immunoreactivity in double-labelled somata was calculated and expressed as average \pm SEM. Co-localization coefficients between secretagogin, parvalbumin, calbindin and calretinin were measured and calculated along the same principle, including animal number and the quantitative attributes of image analysis. We emphasize that this approach was an investigative method and did not aim to provide accurate results regarding cell numbers/proportions.

\section{Human tissue preparation and immunohistochemistry}

We applied direct perfusion via the internal carotid and vertebral arteries, which facilitated the preservation of tissue integrity relative to alternative fixation methods. Human brains $(n=2$, gender and age: female/83 years and male/79 years, with a clinical history lacking neurodegenerative disease, ethical approval: TUKEB 84/2014, Hungary) were first perfused with physiological saline, followed by a fixative containing $2 \%$ PFA and $0.1 \%$ glutaraldehyde in $0.1 \mathrm{M}$ Tris-buffered saline (TBS, pH 7.4) $7 \mathrm{~h}$ or $11 \mathrm{~h}$ after death. The removal and subsequent preparation of human tissues were in accordance with the relevant ethical guidelines of Semmelweis University (1998, Budapest, Hungary). Blocks from the medulla oblongata and pons were dissected out and post-fixed in 2\% PFA in TBS for 72, followed by immersion in cryoprotective $30 \%$ sucrose in $0.1 \mathrm{M} \mathrm{PB}(\mathrm{pH}$ 7.4) overnight. Coronal sections $(50 \mu \mathrm{m})$ were cut on a cryostat microtome and processed for immunohistochemistry. Free-floating sections were rinsed in $\mathrm{PB}(\mathrm{pH} 7.4)$ and pretreated with $0.3 \%$ Triton $\mathrm{X}-100$ (in PB) for $1 \mathrm{~h}$ at $22-24{ }^{\circ} \mathrm{C}$ to enhance the penetration of antibodies. Non-specific immunoreactivity was suppressed by incubating our specimens in a cocktail of 5\% NDS (Jackson), 10\% BSA (Sigma) and $0.3 \%$ Triton X-100 (Sigma) in PB for $1 \mathrm{~h}$ at $22-24{ }^{\circ} \mathrm{C}$. Sections were exposed for up to $72 \mathrm{~h}$ (at $4{ }^{\circ} \mathrm{C}$ ) to the cocktail of primary antibodies (Table 1) diluted in PB to which $0.1 \%$ NDS and $0.3 \%$ Triton X-100 had been added. After extensive rinsing in $\mathrm{PB}$, the immunoreactivities were revealed by chromogenic staining (as above) or by Cy2, 3 or 5-tagged secondary antibodies raised in donkey (1:200 [Jackson], $2 \mathrm{~h}$ at $\left.22-24{ }^{\circ} \mathrm{C}\right)$. Lipofuscin autofluorescence was quenched by applying Sudan Black-B [1\%, dissolved in 70\% ethanol (Schnell et al. 1999)]. Glass-mounted sections were coverslipped with Aquamount embedding medium (Dako). Sections were inspected and images acquired on a 710LSM confocal laser-scanning microscope (Zeiss) at $10 \times$ or $40 \times$ primary magnification and pinhole settings limiting signal detection to 0.5-0.7 $\mu \mathrm{m}$. Emission spectra for each dye were limited as follows: Cy2/505-530 nm, Cy3/560-610 nm, and Cy5/650-720 nm. Multi-panel figures were assembled in CorelDraw X7 (Corel Corp.).

Samples from patients with Alzheimer's disease and agematched controls (without clinical signs of neuropsychiatric disease) were acquired at the Brain Bank of the Institute of Neurology, Medical University of Vienna, Austria, and from the Human Brain Bank of Semmelweis University, Budapest, Hungary (a total of 34 samples were used, for 
Table 2 Demography and use of human subjects

\begin{tabular}{|c|c|c|c|c|}
\hline Case ID & Stage & Age (year) & Gender & Analysis \\
\hline \#130-03 & Braak I-II & 78 & Male & $\mathrm{IHC}$ \\
\hline \#84-03 & Braak I-II & 77 & Male & $\mathrm{IHC}$ \\
\hline \#129-03 & Braak I-II & 77 & Female & IHC \\
\hline$\# 15-05$ & Braak I-II & 78 & Female & IHC \\
\hline \#126-05 & Braak I-II & 80 & Female & IHC \\
\hline \#14-05 & Braak III-IV & 78 & Male & $\mathrm{IHC}$ \\
\hline \#156-03 & Braak III-IV & 77 & Male & $\mathrm{IHC}$ \\
\hline \#131-05 & Braak III-IV & 79 & Female & IHC \\
\hline$\# 58-06$ & Braak III-IV & 80 & Female & $\mathrm{IHC}$ \\
\hline \#100-07 & Braak III-IV & 81 & Male & IHC \\
\hline$\# 9-10$ & Braak V-VI & 84 & Male & IHC \\
\hline \#124-07 & Braak V-VI & 81 & Female & IHC \\
\hline \#88-05 & Braak V-VI & 78 & Male & IHC \\
\hline \#103-06 & Braak V-VI & 81 & Female & IHC \\
\hline \#107-10 & Braak V-VI & 85 & Female & IHC \\
\hline$\# 1$ & Braak I & 63 & Male & qPCR \\
\hline$\# 90$ & Braak I & 78 & Female & qPCR \\
\hline \#98 & Braak I & 74 & Male & qPCR \\
\hline$\# 164$ & Braak I & 85 & Male & $\mathrm{qPCR}$ \\
\hline \#165 & Braak I & 88 & Female & $\mathrm{qPCR}$ \\
\hline$\# 187$ & Braak I & 62 & Male & qPCR \\
\hline$\# 189$ & Braak I & 79 & Female & $\mathrm{qPCR}$ \\
\hline \#220 & Braak I-II & 63 & Male & $\mathrm{qPCR}$ \\
\hline$\# 90$ & Braak I & 78 & Female & $\mathrm{qPCR}$ \\
\hline \#98 & Braak I & 74 & Male & qPCR \\
\hline \#164 & Braak I & 85 & Male & $\mathrm{qPCR}$ \\
\hline$\# 165$ & Braak I & 88 & Female & qPCR \\
\hline \#187 & Braak I & 62 & Male & qPCR \\
\hline$\# 189$ & Braak I & 79 & Female & $\mathrm{qPCR}$ \\
\hline \#220 & Braak I-II & 63 & Male & qPCR \\
\hline \#94 & Braak III-IV & 65 & Male & qPCR \\
\hline \#185 & Braak III-IV & 80 & Male & qPCR \\
\hline \#196 & Braak III-IV & 78 & Female & qPCR \\
\hline \#197 & Braak III-IV & 64 & Male & $\mathrm{qPCR}$ \\
\hline \#222 & Braak III-IV & 80 & Male & qPCR \\
\hline \#163 & Braak V-VI & 81 & Female & qPCR \\
\hline \#177 & Braak V-VI & 89 & Female & qPCR \\
\hline \#188 & Braak V-VI & 86 & Female & qPCR \\
\hline \#192 & Braak V-VI & 81 & Male & qPCR \\
\hline \#195 & Braak V-VI & 83 & Male & $\mathrm{qPCR}$ \\
\hline \#201 & Braak V-VI & 74 & Male & qPCR \\
\hline \#185 & Braak III-IV & 80 & Male & qPCR \\
\hline \#196 & Braak III-IV & 78 & Female & qPCR \\
\hline \#197 & Braak III-IV & 64 & Male & $\mathrm{qPCR}$ \\
\hline \#222 & Braak III-IV & 80 & Male & $\mathrm{qPCR}$ \\
\hline \#163 & Braak V-VI & 81 & Female & qPCR \\
\hline \#177 & Braak V-VI & 89 & Female & $\mathrm{qPCR}$ \\
\hline \#188 & Braak V-VI & 86 & Female & $\mathrm{qPCR}$ \\
\hline \#192 & Braak V-VI & 81 & Male & qPCR \\
\hline \#195 & Braak V-VI & 83 & Male & qPCR \\
\hline
\end{tabular}

Table 2 (continued)

\begin{tabular}{lllll}
\hline Case ID & Stage & Age (year) & Gender & Analysis \\
\hline \#201 & Braak V-VI & 74 & Male & qPCR \\
\hline
\end{tabular}

details see Table 2). For immunohistochemistry, pontine blocks including the locus coeruleus were immersion fixed in 4\% PFA in PB for 2-3 weeks at room temperature. These cases had previously been characterized for Alzheimer's disease-related pathologies (amyloid $\beta(\mathrm{A} \beta$ ) plaque burden, intracellular tau accumulation) and other neurodegenerative conditions had been excluded. Tissues were obtained and used compliant with the Declaration of Helsinki and following institutional guidelines. The study was performed in the course of an approved study by the Ethical Committee of the Medical University of Vienna (No.1454/2018). All patient material was coded to ensure anonymity throughout tissue processing. For real-time quantitative PCR experiments, locus coeruleus micropunches from human samples were homogenized in isolating buffer (Qiagen). RNA was extracted using the RNeasy mini kit (Qiagen) with a DNase I step performed to eliminate traces of genomic DNA30 and reverse transcribed using a high-capacity cDNA reverse transcription kit (Applied Biosystems). Reactions were performed after an initial denaturation at $95{ }^{\circ} \mathrm{C}$ for $10 \mathrm{~min}$, followed by 40 cycles of $95^{\circ} \mathrm{C}$ for $15 \mathrm{~s}$ denaturation, annealing and extension at calculated temperatures $(60 \mathrm{~s})$ and a dissociation stage (from 60 to $95{ }^{\circ} \mathrm{C}$ with $0.5{ }^{\circ} \mathrm{C}$ steps for $10 \mathrm{~s}$ each; CFX96, Bio-Rad), with primer pairs amplifying short fragments for the secretagogin gene (forward: 5'-CTGTTAGATGGCTCTGCCTGTC-3', reverse: 5'-GTT ACAGGATTGCCATGAATGC-3'). Samples without template or reverse transcriptase served as negative controls. Expression levels were normalized to the housekeeping gene encoding glyceraldehyde-3-phosphate dehydrogenase (GAPDH, forward: 5'-AACTTTGGCATTGTGGAAGG-3', reverse: 5'ACACATTGGGGGTAGGAACA3') obtained for every sample in parallel assays. Quantitative (q)PCR was performed on a Bio-Rad CFX 96 thermal cycler. Samples were run in triplicate to avoid processing-related deviations.

\section{Whole mounts}

Neural tissue explants were isolated from E8.5 and E11.5 mouse embryos from pregnant mice ( $n=4$ in total) with a modified technique described elsewhere (Echevarria et al. 2001). Briefly, neural tubes were opened by cutting the roof plate on ice-cold PBS. Once flattened as an open book, they were placed on Millicell cell culture plate inserts (Millipore, pore size $0.4 \mu \mathrm{m}$ ) and cultured for 3 days as on Neurobasal medium penicillin $(100 \mathrm{U} / \mathrm{mL})$, and streptomycin $(100 \mu \mathrm{g} /$ $\mathrm{mL}$ ) (all from Invitrogen) in an incubator at $37^{\circ} \mathrm{C}$, with $5 \% \mathrm{CO}_{2}$ and $95 \%$ humidity. The neural tube explants were 
subsequently removed from the membranes, fixed in PFA4\% and immunostained as described above.

\section{Statistical analysis}

Data were analysed using Statistical Package for the Social Sciences (version 17.0, SPSS Inc.). Immunofluorescence intensities were evaluated using Student's $t$ test (on independent samples, $\left.{ }^{*} p<0.05\right)$. qPCR data were analysed using one-way ANOVA with Tukey's post hoc tests. Data were expressed as mean \pm SEM. A $p$ value of $<0.05$ was considered statistically significant.

Acknowledgements Open access funding provided by Semmelweis University (SE). We would like to thank M. Celio for his advice and providing us with immunohistochemical images (Fig. 3). This work was supported by the National Brain Research Program of Hungary (2017-1.2.1-NKP2017-00002,A.A.); Excellence Program for Higher Education of Hungary (FIKP-2018, A.A.); Swedish Research Council (T.G.M.H., T.H.); Novo Nordisk Foundation (T.G.M.H.,T.H.); Hjärnfonden (T.H.); European Research Council (SECRET-CELLS, 2015-AdG-695136; T.H.) and intramural funds of the Medical University of Vienna (T.H.).

\section{Compliance with ethical standards}

Conflict of interest The authors declare no conflict of interest.

Ethical approval All procedures performed in studies involving human participants were in accordance with the ethical standards of the national research committee of Hungary (TUKEB 84/2014) and with the Helsinki Declaration and its later amendments or comparable ethical standards. All applicable international, national and institutional guidelines for the care and use of animals were followed.

Open Access This article is distributed under the terms of the Creative Commons Attribution 4.0 International License (http://creativeco mmons.org/licenses/by/4.0/), which permits unrestricted use, distribution, and reproduction in any medium, provided you give appropriate credit to the original author(s) and the source, provide a link to the Creative Commons license, and indicate if changes were made.

\section{References}

Alloul K, Sauriol L, Kennedy W, Laurier C, Tessier G, Novosel S, Contandriopoulos A (1998) Alzheimer's disease: a review of the disease, its epidemiology and economic impact. Arch Gerontol Geriatr 27:189-221

Alpar A, Gartner U, Seeger G, Hartig W, Brauer K, Arendt T (2004) Constitutive expression of $\mathrm{p} 21 \mathrm{H}$-ras(Val12) in pyramidal neurons results in reorganization of mouse neocortical afferents. J Neurobiol 60:263-274

Alpar A, Kunzle H, Gartner U, Popkova Y, Bauer U, Grosche J, Reichenbach A, Hartig W (2010) Slow age-dependent decline of doublecortin expression and BrdU labeling in the forebrain from lesser hedgehog tenrecs. Brain Res 1330:9-19

Alpar A, Attems J, Mulder J, Hokfelt T, Harkany T (2012) The renaissance of $\mathrm{Ca} 2+$-binding proteins in the nervous system: secretagogin takes center stage. Cell Signal 24:378-387
Alpar A, Zahola P, Hanics J, Hevesi Z, Korchynska S, Benevento M, Pifl C, Zachar G, Perugini J, Severi I, Leitgeb P, Bakker J, Miklosi AG, Tretiakov E, Keimpema E, Arque G, Tasan RO, Sperk G, Malenczyk K, Mate Z et al (2018) Hypothalamic CNTF volume transmission shapes cortical noradrenergic excitability upon acute stress. EMBO J. https://doi.org/10.15252/embj.2018100087

Andressen C, Blumcke I, Celio MR (1993) Calcium-binding proteins: selective markers of nerve cells. Cell Tissue Res 271:181-208

Aston-Jones G, Rajkowski J, Kubiak P, Valentino RJ, Shipley MT (1996) Role of the locus coeruleus in emotional activation. Prog Brain Res 107:379-402

Attems J, Preusser M, Grosinger-Quass M, Wagner L, Lintner F, Jellinger K (2008) Calcium-binding protein secretagogin-expressing neurones in the human hippocampus are largely resistant to neurodegeneration in Alzheimer's disease. Neuropathol Appl Neurobiol 34:23-32

Attems J, Ittner A, Jellinger K, Nitsch RM, Maj M, Wagner L, Gotz J, Heikenwalder M (2011) Reduced secretagogin expression in the hippocampus of P301L tau transgenic mice. J Neural Transm 118:737-745

Attems J, Alpar A, Spence L, McParland S, Heikenwalder M, Uhlen M, Tanila H, Hokfelt TG, Harkany T (2012a) Clusters of secretagogin-expressing neurons in the aged human olfactory tract lack terminal differentiation. Proc Natl Acad Sci USA 109:6259-6264

Attems J, Thomas A, Jellinger K (2012b) Correlations between cortical and subcortical tau pathology. Neuropathol Appl Neurobiol 38:582-590

Barde S, Ruegg J, Prud'homme J, Ekstrom TJ, Palkovits M, Turecki G, Bagdy G, Ihnatko R, Theodorsson E, Juhasz G, Diaz-Heijtz R, Mechawar N, Hokfelt TG (2016) Alterations in the neuropeptide galanin system in major depressive disorder involve levels of transcripts, methylation, and peptide. Proc Natl Acad Sci USA 113:E8472-E8481

Barker DA, Dreher B (1998) Spatiotemporal patterns of ontogenetic expression of parvalbumin in the superior colliculi of rats and rabbits. J Comp Neurol 393:210-230

Bastianelli E, Pochet R (1993) Transient expression of calretinin during development of chick cerebellum. Comparison with calbindinD28k. Neurosci Res 17:53-61

Bauer MC, O'Connell DJ, Maj M, Wagner L, Cahill DJ, Linse S (2011) Identification of a high-affinity network of secretagogin-binding proteins involved in vesicle secretion. Mol BioSyst 7:2196-2204

Boscia F, Casamassa A, Secondo A, Esposito A, Pannaccione A, Sirabella R, Pignataro G, Cuomo O, Vinciguerra A, de Rosa V, Annunziato L (2016) NCX1 exchanger cooperates with calretinin to confer preconditioning-induced tolerance against cerebral ischemia in the striatum. Mol Neurobiol 53:1365-1376

Braak H, Del Tredici K (2012) Where, when, and in what form does sporadic Alzheimer's disease begin? Curr Opin Neurol 25:708-714

Bremner JD, Krystal JH, Southwick SM, Charney DS (1996a) Noradrenergic mechanisms in stress and anxiety: I. Preclinical studies. Synapse 23:28-38

Bremner JD, Krystal JH, Southwick SM, Charney DS (1996b) Noradrenergic mechanisms in stress and anxiety: II. Clinical studies. Synapse 23:39-51

Celio MR (1990) Calbindin D-28k and parvalbumin in the rat nervous system. Neuroscience 35:375-475

Chan-Palay V, Asan E (1989) Alterations in catecholamine neurons of the locus coeruleus in senile dementia of the Alzheimer type and in Parkinson's disease with and without dementia and depression. J Comp Neurol 287:373-392

Charney DS (2003) Neuroanatomical circuits modulating fear and anxiety behaviors. Acta Psychiatr Scand Suppl 417:38-50

Dahlstroem A, Fuxe K (1964) Evidence for the existence of monoamine-containing neurons in the central nervous system. I. 
Demonstration of monoamines in the cell bodies of brain stem neurons. Acta Physiol Scand Suppl 232:1-55

Davern PJ (2014) A role for the lateral parabrachial nucleus in cardiovascular function and fluid homeostasis. Front Physiol 5:436

de Haan W, van Straaten ECW, Gouw AA, Stam CJ (2017) Altering neuronal excitability to preserve network connectivity in a computational model of Alzheimer's disease. PLoS Comput Biol 13:e1005707

del Rio MR, DeFelipe J (1997) Colocalization of parvalbumin and calbindin D-28k in neurons including chandelier cells of the human temporal neocortex. J Chem Neuroanat 12:165-173

Echevarria D, Vieira C, Martinez S (2001) Mammalian neural tube grafting experiments: an in vitro system for mouse experimental embryology. Int J Dev Biol 45:895-902

Enderlin S, Norman AW, Celio MR (1987) Ontogeny of the calcium binding protein calbindin D-28k in the rat nervous system. Anat Embryol 177:15-28

Faull OK, Subramanian HH, Ezra M, Pattinson KTS (2019) The midbrain periaqueductal gray as an integrative and interoceptive neural structure for breathing. Neurosci Biobehav Rev 98:135-144

Freund TF, Buzsáki G (1996) Interneurons of the hippocampus. Hippocampus $6: 347-470$

Freund TF, Buzsaki G, Leon A, Baimbridge KG, Somogyi P (1990) Relationship of neuronal vulnerability and calcium binding protein immunoreactivity in ischemia. Exp Brain Res 83:55-66

Friauf E (1993) Transient appearance of calbindin-D28k-positive neurons in the superior olivary complex of developing rats. $J$ Comp Neurol 334:59-74

Garas FN, Shah RS, Kormann E, Doig NM, Vinciati F, Nakamura KC, Dorst MC, Smith Y, Magill PJ, Sharott A (2016) Secretagogin expression delineates functionally-specialized populations of striatal parvalbumin-containing interneurons. Elife 5:e16088

Gartner W, Vila G, Daneva T, Nabokikh A, Koc-Saral F, Ilhan A, Majdic O, Luger A, Wagner L (2007) New functional aspects of the neuroendocrine marker secretagogin based on the characterization of its rat homolog. Am J Physiol Endocrinol Metab 293:E347-E354

Gati G, Lendvai D, Hokfelt T, Harkany T, Alpar A (2014) Revival of calcium-binding proteins for neuromorphology: secretagogin typifies distinct cell populations in the avian brain. Brain Behav Evol 83:82-92

German DC, Manaye KF, White CL 3rd, Woodward DJ, McIntire DD, Smith WK, Kalaria RN, Mann DM (1992) Disease-specific patterns of locus coeruleus cell loss. Ann Neurol 32:667-676

Gyengesi E, Andrews ZB, Paxinos G, Zaborszky L (2013) Distribution of secretagogin-containing neurons in the basal forebrain of mice, with special reference to the cholinergic corticopetal system. Brain Res Bull 94:1-8

Hanics J, Szodorai E, Tortoriello G, Malenczyk K, Keimpema E, Lubec G, Hevesi Z, Lutz MI, Kozsurek M, Puskar Z, Toth ZE, Wagner L, Kovacs GG, Hokfelt TG, Harkany T, Alpar A (2017) Secretagogin-dependent matrix metalloprotease-2 release from neurons regulates neuroblast migration. Proc Natl Acad Sci USA 114:E2006-E2015

Hokfelt T, Barde S, Xu ZD, Kuteeva E, Ruegg J, Le Maitre E, Risling M, Kehr J, Ihnatko R, Theodorsson E, Palkovits M, Deakin W, Bagdy G, Juhasz G, Prud'homme HJ, Mechawar N, Diaz-Heijtz R, Ogren SO (2018) Neuropeptide and small transmitter coexistence: fundamental studies and relevance to mental illness. Front Neural Circuits 12:106

Holmes A, Picciotto MR (2006) Galanin: a novel therapeutic target for depression, anxiety disorders and drug addiction? CNS Neurol Disord Drug Targets 5:225-232

Itoi K, Sugimoto N (2010) The brainstem noradrenergic systems in stress, anxiety and depression. J Neuroendocrinol 22:355-361
Jacobowitz DM, Winsky L (1991) Immunocytochemical localization of calretinin in the forebrain of the rat. J Comp Neurol 304:198-218

Kelly SC, He B, Perez SE, Ginsberg SD, Mufson EJ, Counts SE (2017) Locus coeruleus cellular and molecular pathology during the progression of Alzheimer's disease. Acta Neuropathol Commun 5:8

Kosaka K, Kosaka T (2013) Secretagogin-containing neurons in the mouse main olfactory bulb. Neurosci Res 77:16-32

Kosaka T, Kosaka K (2018) Calcium-binding protein, secretagogin, specifies the microcellular tegmental nucleus and intermediate and ventral parts of the cuneiform nucleus of the mouse and rat. Neurosci Res 134:30-38

Kosaka T, Yasuda S, Kosaka K (2017) Calcium-binding protein, secretagogin, characterizes novel groups of interneurons in the rat striatum. Neurosci Res 119:53-60

Kraner SD, Norris CM (2018) Astrocyte activation and the calcineurin/ NFAT pathway in cerebrovascular disease. Front Aging Neurosci 10:287

Kuteeva E, Hokfelt T, Wardi T, Ogren SO (2010) Galanin, galanin receptor subtypes and depression-like behaviour. EXS 102:163-181

Kvetnansky R, Sabban EL, Palkovits M (2009) Catecholaminergic systems in stress: structural and molecular genetic approaches. Physiol Rev 89:535-606

Lee EC, Yu D, Martinez de Velasco J, Tessarollo L, Swing DA, Court DL, Jenkins NA, Copeland NG (2001) A highly efficient Escherichia coli-based chromosome engineering system adapted for recombinogenic targeting and subcloning of BAC DNA. Genomics 73:56-65

Lendvai D, Morawski M, Negyessy L, Gati G, Jager C, Baksa G, Glasz T, Attems J, Tanila H, Arendt T, Harkany T, Alpar A (2013) Neurochemical mapping of the human hippocampus reveals perisynaptic matrix around functional synapses in Alzheimer's disease. Acta Neuropathol 125:215-229

Ljungdahl A, Hokfelt T, Nilsson G (1978) Distribution of substance P-like immunoreactivity in the central nervous system of the rat-I. Cell bodies and nerve terminals. Neuroscience 3:861-943

Lohmann C, Friauf E (1996) Distribution of the calcium-binding proteins parvalbumin and calretinin in the auditory brainstem of adult and developing rats. J Comp Neurol 367:90-109

Lu X, Sharkey L, Bartfai T (2007) The brain galanin receptors: targets for novel antidepressant drugs. CNS Neurol Disord: Drug Targets 6:183-192

Ludwig M, Leng G (2006) Dendritic peptide release and peptidedependent behaviours. Nat Rev Neurosci 7:126-136

Malenczyk K, Girach F, Szodorai E, Storm P, Segerstolpe A, Tortoriello G, Schnell R, Mulder J, Romanov RA, Borok E, Piscitelli F, Di Marzo V, Szabo G, Sandberg R, Kubicek S, Lubec G, Hokfelt T, Wagner L, Groop L, Harkany T (2017) A TRPV1-to-secretagogin regulatory axis controls pancreatic beta-cell survival by modulating protein turnover. EMBO J 36:2107-2125

Manns M, Gunturkun O (2003) Light experience induces differential asymmetry pattern of GABA- and parvalbumin-positive cells in the pigeon's visual midbrain. J Chem Neuroanat 25:249-259

Markia B, Kovacs ZI, Palkovits M (2008) Projections from the vestibular nuclei to the hypothalamic paraventricular nucleus: morphological evidence for the existence of a vestibular stress pathway in the rat brain. Brain Struct Funct 213:239-245

Martins I, Tavares I (2017) Reticular formation and pain: the past and the future. Front Neuroanat 11:51

McHaffie JG, Anstrom KK, Gabriele ML, Stein BE (2001) Distribution of the calcium-binding proteins calbindin D-28k and parvalbumin in the superior colliculus of adult and neonatal cat and rhesus monkey. Exp Brain Res 141:460-470

Morrison SF, Nakamura K (2011) Central neural pathways for thermoregulation. Front Biosci (Landmark Ed) 16:74-104 
Mulder J, Zilberter M, Spence L, Tortoriello G, Uhlen M, Yanagawa Y, Aujard F, Hökfelt T, Harkány T (2009) Secretagogin is a Ca2+binding protein specifying subpopulations of telencephalic neurons. Proc Natl Acad Sci USA 106:22492-22497

Mulder J, Spence L, Tortoriello G, Dinieri JA, Uhlen M, Shui B, Kotlikoff MI, Yanagawa Y, Aujard F, Hokfelt T, Hurd YL, Harkany $\mathrm{T}$ (2010) Secretagogin is a Ca2+-binding protein identifying prospective extended amygdala neurons in the developing mammalian telencephalon. Eur J Neurosci 31:2166-2177

Palkovits M (1999) Interconnections between the neuroendocrine hypothalamus and the central autonomic system. Geoffrey Harris Memorial Lecture, Kitakyushu, Japan, October 1998. Front Neuroendocrinol 20:270-295

Parra-Damas A, Saura CA (2019) Synapse-to-nucleus signaling in neurodegenerative and neuropsychiatric disorders. Biol Psychiatry. https://doi.org/10.1016/j.biopsych.2019.01.006

Porteros A, Brinon JG, Arevalo R, Crespo C, Aijon J, Alonso JR (1998) Transient expression of calretinin in the trout habenulo-interpeduncular system during development. Neurosci Lett 254:9-12

Puyal J, Devau G, Venteo S, Sans N, Raymond J (2002) Calciumbinding proteins map the postnatal development of rat vestibular nuclei and their vestibular and cerebellar projections. J Comp Neurol 451:374-391

Rees CL, White CM, Ascoli GA (2017) Neurochemical markers in the mammalian brain: structure, roles in synaptic communication, and pharmacological relevance. Curr Med Chem 24:3077-3103

Riedel A, Hartig W, Seeger G, Gartner U, Brauer K, Arendt T (2002) Principles of rat subcortical forebrain organization: a study using histological techniques and multiple fluorescence labeling. J Chem Neuroanat 23:75-104

Rogstam A, Linse S, Lindqvist A, James P, Wagner L, Berggard T (2007) Binding of calcium ions and SNAP-25 to the hexa EF-hand protein secretagogin. Biochem J 401:353-363

Romanov RA, Alpar A, Zhang MD, Zeisel A, Calas A, Landry M, Fuszard M, Shirran SL, Schnell R, Dobolyi A, Olah M, Spence L, Mulder J, Martens H, Palkovits M, Uhlen M, Sitte HH, Botting $\mathrm{CH}$, Wagner L, Linnarsson S et al (2015) A secretagogin locus of the mammalian hypothalamus controls stress hormone release. EMBO J 34:36-54

Rostovskaya M, Fu J, Obst M, Baer I, Weidlich S, Wang H, Smith AJ, Anastassiadis K, Stewart AF (2012) Transposon-mediated BAC transgenesis in human ES cells. Nucleic Acids Res 40:e150

Sabbir MG (2018) Loss of $\mathrm{Ca}(2+) /$ calmodulin dependent protein kinase kinase 2 leads to aberrant transferrin phosphorylation and trafficking: a potential biomarker for Alzheimer's disease. Front Mol Biosci 5:99

Samuels ER, Szabadi E (2008a) Functional neuroanatomy of the noradrenergic locus coeruleus: its roles in the regulation of arousal and autonomic function part I: principles of functional organisation. Curr Neuropharmacol 6:235-253

Samuels ER, Szabadi E (2008b) Functional neuroanatomy of the noradrenergic locus coeruleus: its roles in the regulation of arousal and autonomic function part II: physiological and pharmacological manipulations and pathological alterations of locus coeruleus activity in humans. Curr Neuropharmacol 6:254-285
Schnell SA, Staines WA, Wessendorf MW (1999) Reduction of lipofuscin-like autofluorescence in fluorescently labeled tissue. J Histochem Cytochem 47:719-730

Skelton NJ, Kordel J, Akke M, Forsen S, Chazin WJ (1994) Signal transduction versus buffering activity in $\mathrm{Ca}(2+)$-binding proteins. Nat Struct Biol 1:239-245

Sompol P, Norris CM (2018) $\mathrm{Ca}(2+)$, astrocyte activation and calcineurin/NFAT signaling in age-related neurodegenerative diseases. Front Aging Neurosci 10:199

Takizawa C, Thompson PL, van Walsem A, Faure C, Maier WC (2015) Epidemiological and economic burden of Alzheimer's disease: a systematic literature review of data across Europe and the United States of America. J Alzheimer's Dis 43:1271-1284

Tomlinson BE, Irving D, Blessed G (1981) Cell loss in the locus coeruleus in senile dementia of Alzheimer type. J Neurol Sci 49:419-428

Verdaguer E, Brox S, Petrov D, Olloquequi J, Romero R, de Lemos ML, Camins A, Auladell C (2015) Vulnerability of calbindin, calretinin and parvalbumin in a transgenic/knock-in APPswe/PS1dE9 mouse model of Alzheimer disease together with disruption of hippocampal neurogenesis. Exp Gerontol 69:176-188

Vila-Porcile E, Xu ZQ, Mailly P, Nagy F, Calas A, Hokfelt T, Landry M (2009) Dendritic synthesis and release of the neuropeptide galanin: morphological evidence from studies on rat locus coeruleus neurons. J Comp Neurol 516:199-212

Wagner L, Oliyarnyk O, Gärtner W, Nowotny P, Groeger M, Kaserer K, Waldhausl W, Pasternack MS (2000) Cloning and expression of secretagogin, a novel neuroendocrine- and pancreatic islet of Langerhans-specific Ca2+-binding protein. J Biol Chem 275:24740-24751

Wild JM (1981) Identification and localization of the motor nuclei and sensory projections of the glossopharyngeal, vagus, and hypoglossal nerves of the cockatoo (Cacatua roseicapilla), Cacatuidae. J Comp Neurol 203:351-377

Wouterlood FG, Grosche J, Hartig W (2001) Co-localization of calretinin and calbindin in distinct cells in the hippocampal formation of the rat. Brain Res 922:310-314

Yi H, Bao X, Tang X, Fan X, Xu H (2016) Estrogen modulation of calretinin and BDNF expression in midbrain dopaminergic neurons of ovariectomised mice. J Chem Neuroanat 77:60-67

Zaborszky L, Beinfeld MC, Palkovits M, Heimer L (1984) Brainstem projection to the hypothalamic ventromedial nucleus in the rat: a CCK-containing long ascending pathway. Brain Res 303:225-231

Zhang MD, Barde S, Szodorai E, Josephson A, Mitsios N, Watanabe M, Attems J, Lubec G, Kovacs GM, Uhlen M, Mulder J, Harkany T, Hokfelt T (2016) Comparative anatomical distribution of neuronal calcium-binding protein (NECAB) 1 and -2 in rodent and human spinal cord. Brain Struct Funct 221:3803-3823

Publisher's Note Springer Nature remains neutral with regard to jurisdictional claims in published maps and institutional affiliations. 